\title{
Recent DIII-D Advances in Runaway Electron Measurement and Model Validation
}

C. Paz-Soldan, ${ }^{1}$ N. W. Eidietis,${ }^{1}$ E. M. Hollmann, ${ }^{2}$ P. Aleynikov, ${ }^{3}$ L. Carbajal, ${ }^{4}$ W. W. Heidbrink ${ }^{5}$, M. Hoppe ${ }^{6}$, C. Liu, ${ }^{7}$ A. Lvovskiy, ${ }^{8}$ D. Shiraki, ${ }^{4}$ D. Spong, ${ }^{4}$ D. P. Brennan, ${ }^{9}$ C. M. Cooper,${ }^{8}$ D. del-Castillo-Negrete, ${ }^{4}$ X. Du, ${ }^{1}$ O. Embreus, ${ }^{6}$ T. Fulop, ${ }^{6}$ J. Herfindal, ${ }^{4}$ R. Moyer ${ }^{2}$ P. Parks,${ }^{1}$ and K. E. Thome ${ }^{1}$

${ }^{1}$ General Atomics, San Diego, CA 92186-5608, USA

${ }^{2}$ University of California San Diego, La Jolla, CA 92093-0417, USA

${ }^{3}$ Max-Planck Institute for Plasma Physics, Greifswald, Germany

${ }^{4}$ Oak Ridge National Laboratory, Oak Ridge, TN 37831, USA

${ }^{5}$ University of California Irvine, Irvine, CA 92697, USA

${ }^{6}$ Chalmers University of Technology, Goteborg, Sweden

${ }^{7}$ Princeton Plasma Physics Laboratory, Princeton, New Jersey 08543-0451, USA

${ }^{8}$ Oak Ridge Associated Universities, Oak Ridge, TN 37831, USA

${ }^{9}$ Princeton University, Princeton, New Jersey 08543-0451, USA

\begin{abstract}
Novel measurements and modeling of runaway electron (RE) dynamics in DIII-D have resolved experimental discrepancies and validated predictions for ITER, improving confidence that RE avoidance and mitigation can be predictably achieved. Considering RE formation, first experimental assessments of the RE seed current demonstrates that present hot-tail theories are not yet accurate and require improved treatment of the pellet dynamics. Novel measurements of kinetic instabilities in the MHz-range have been made in the RE formation phase, with the intensity of these modes correlated with previously unexplained empirical thresholds for RE generation. Controlled RE dissipation experiments in quiescent regimes have validated $\mathrm{RE}$ distribution function dependencies on collisional and synchrotron damping, both in terms of distribution function shape and dissipation rates. Measurements of RE bremsstrahlung and synchrotron emission are now used in tandem to resolve energy and pitch-angle effects. A resolution to long-standing dissipation anomalies in the quiescent regime is offered by taking into account kinetic instability effects on RE phase-space dynamics. Kinetic instabilities in the $100-200 \mathrm{MHz}$ range are directly observed, though modeling finds the largest dissipation arises from $\mathrm{GHz}$ range instabilities that are beyond the reach of existing diagnostics. Kinetic instabilities are also observed in the mature post-disruption RE plateau phase, so long as the collisional damping rate is reduced with low-Z injection. Experiments with high- $Z$ injection find that the dissipation rate saturates with injection quantity, likely due to neutral diffusion rates being slower than vertical instability rates in DIII-D. Considering the final loss, a 0-D model for first-wall Joule heating is found to be in agreement with experiment, and controlled access to RE equilibria with edge safety factor of 2 identifies novel dynamics brought about by large-scale kink instabilities. These dynamics are typified by fast (tens of microseconds) $\mathrm{RE}$ loss rates without $\mathrm{RE}$ beam regeneration. The above measurements and comparison with theory represent significant advances in
\end{abstract}


the understanding of RE dynamics and indicate possible new opportunities for $\mathrm{RE}$ avoidance or mitigation via kinetic instabilities. 


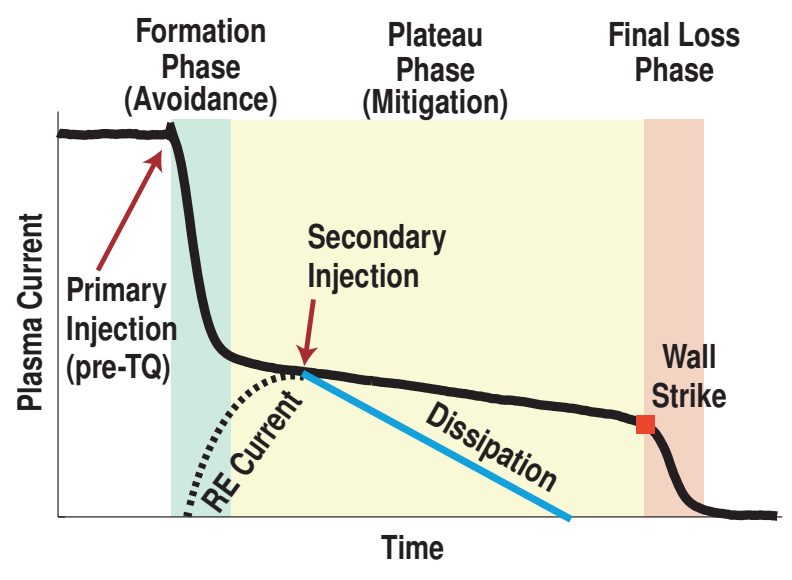

Figure 1. Evolution of a typical disruption including RE formation and loss. This work highlights progress targetting each of the three phases of the RE life-cycle: 1) the formation phase, 2) the mature plateau phase, and 3) the final loss phase.

\section{Introduction}

The runaway electron (RE) problem is of existential concern for fusion-grade tokamaks [1, 2, 3, 4, as the expected RE multiplication rate increases exponentially with plasma current $\left(I_{P}\right)$ [5]. The severe potential for damage from REs, already witnessed in present-day large tokamaks [6], will greatly limit opportunities for empirical tuning of RE mitigation methods. Instead, predictive modeling will be heavily relied upon to optimize RE mitigation for ITER and beyond.

In this work, recent advances in the measurement of RE populations at DIII-D will be summarized with a special focus on theoretical model validation and resulting understanding of the extrapolation to ITER conditions. The RE life-cycle can be divided into three phases as shown in Fig. 1. Each phase has its own challenges to address and will be treated separately, thus forming the structure of this paper.

The first line of defense against the RE threat is avoiding RE generation in the first place, which can be achieved in the short time during and after the thermal quench (TQ). During the TQ, a rapid release of energy gives rise to substantially higher resistivity that rapidly decays the pre-disruption current, giving rise to the current quench (CQ). Simultaneously, the higher resistivity induces large electric fields that accelerate 'seed' REs from the tail of the pre-TQ distribution. During this time, RE avoidance could be achieved by judicious selection of 'primary injection', meant to mitigate the TQ/CQ and possibly also avoid REs. Alternatively, natural processes within the plasma such as magnetic stochasticity [7, 8, 9, 10] and kinetic instabilities of the RE beam [11, 12, 13] may assist in RE avoidance, as will be described.

Barring successful RE avoidance, the mature RE plateau rapidly forms through avalanche multiplication of the initial seed current, potentially carrying a large fraction of the pre-disruption current. This period offers a second opportunity for RE control, namely through the active application of a RE mitigation technique (for example 
through high-Z nobel gas injection for collisional suppression). A successful RE mitigation would dissipate the RE kinetic energy and magnetic energy (RE current) prior to the RE beam coming into contact with the tokamak first-wall. This has been achieved in long-duration RE plateaus under shape control [14, 15, 16], though as will be described, challenges are encountered when the RE beam is vertically unstable [6].

If both RE avoidance and mitigation are unsuccessful, the RE beam will begin to deposit energy into the first-wall and begin the final loss phase. While undesirable in principle there is still a scientific need to understand and predict the impact of this phase, such as to determine the tolerable RE current at the final loss. Effects in this phase thus determine the degree of RE mitigation necessary to avoid damage to the first-wall.

This work will now proceed through each of these phases chronologically and will highlight important recent contributions from both measurements and modeling of RE dynamics observed on DIII-D. The structure of this work is as follows: Section 2 discusses the RE formation phase, Section 3 discusses quiescent (low density Ohmic flat-top) regime experiments simulating RE plateau dissipation, Section 4 discusses postdisruption RE plateau dissipation itself, and Sec. 5 discusses the final loss. Discussion and conclusion are given in Sec. 6. Note some aspects presented in this work have been explored in previous papers; in such cases progress is summarized and new developments appended.

Before embarking, a few unifying themes are identified. The first is new dynamics arising from instability. Kinetic instabilities (in the few $\mathrm{MHz}$ or $100-200 \mathrm{MHz}$ range) have been excited and measured across a breadth of DIII-D RE conditions. Theoretical modeling including these instabilities finds a resolution to long-standing dissipation anomalies in quiescent scenarios, and kinetic instability energy is correlated with empirically observed thresholds for RE generation in disruptive scenarios. Further, bursty magnetohydrodynamic (MHD) instability is observed when the RE current exceeds a threshold corresponding to edge safety factor of 2 , providing an alternate path to the final loss at high current. A second theme is improved experimental measurements enabling more accurate theoretical comparison. Improved measurements of the RE seed current, the RE distribution function, and the RE-induced Joule heating of the firstwall are now available. These advances both improve confidence that RE avoidance and mitigation can be predicted, as well as open new opportunities for RE control - namely via kinetic instability.

\section{RE Formation Phase: Avoidance}

The first phase considered is the time immediately after the TQ and during the CQ when the RE beam is forming. Novel measurements in the TQ allow the first experimental estimate of the RE seed current on DIII-D, with leading theories found to both under and overpredict the measurements by several order of magnitude. Later, during the CQ, $\mathrm{MHz}$-frequency kinetic instabilities are observed during RE avalanche multiplication, 


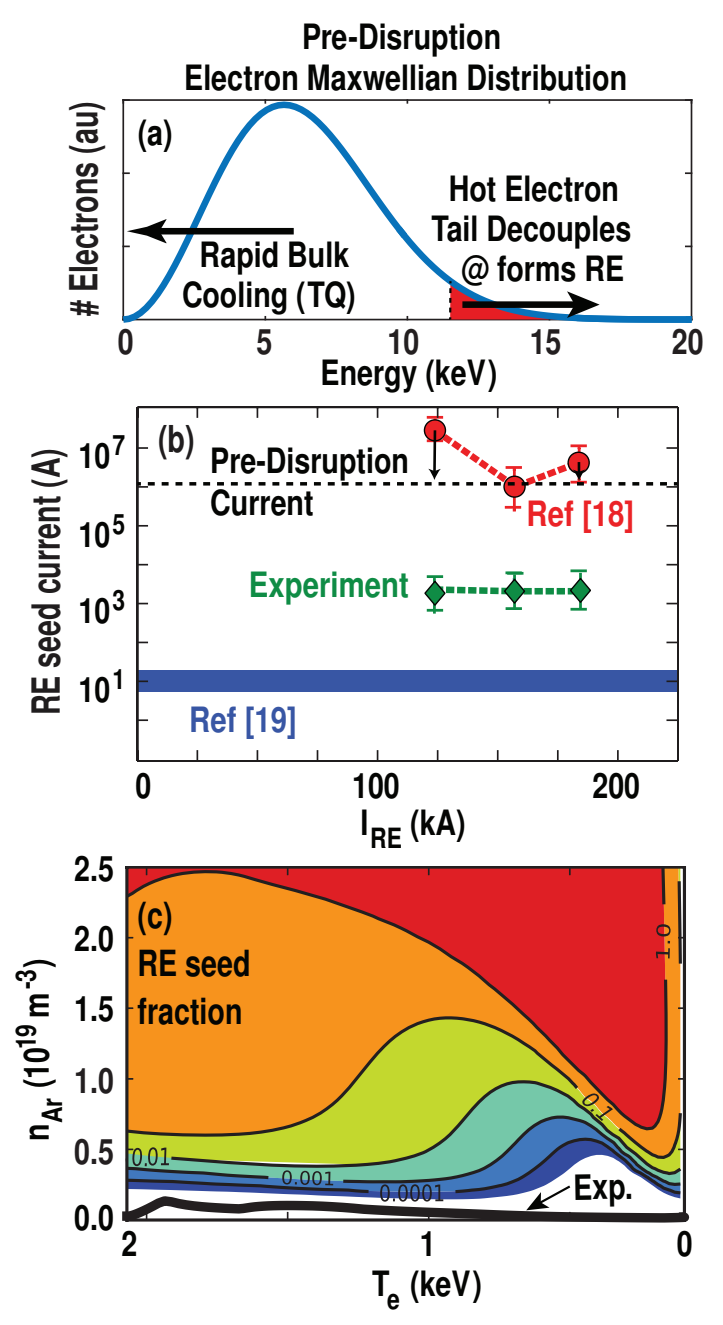

Figure 2. (a) Cartoon depicting 'hot-tail' RE seed formation process. (b) Measurement of RE seed current [17] and comparison to model calculations from Refs. [18] and [19. (c) Ref. [19] model dependence of RE seed fraction on Ar density $\left(n_{A r}\right)$ and pre-TQ $T_{e}$ (proxy for radius) in DIII-D conditions, along with experimental estimates of ablated Ar as a function of $T_{e}$ (mapping from radius).

with instability energy correlated with empirical thresholds for RE formation in terms of plasma current and primary mitigation injection quantity.

\subsection{RE Seed Current Estimate and Prediction}

A novel technique has been developed to estimate the RE seed current from measurements of enhanced ablation of small cryogenic argon (Ar) pellets [17]. These pellets are used to maximize the likelihood of RE production in DIII-D. The technique compares measured Ar-I emission to the expected ablation level from the well-measured thermal plasma profiles. Any excess Ar-I light above these levels is attributed to the RE population further ablating the pellet, yielding an estimate of the RE seed current prior to avalanche multiplication. Importantly, high-velocity pellets were used in this 
study, which due to the faster TQ time maintained the density below the ECE cutoff density (at the measurement location toroidally opposite the pellet). This enabled the use of high temporal resolution $T_{e}$ profiles from ECE during the pellet-induced TQ. Furthermore, high velocity pellets simplified the interpretation of the excess ablation as cross-field transport effects of REs and impurities could be neglected. As shown in Fig. 2 (b), this estimation technique yields RE seed magnitudes of $\mathcal{O}\left(10^{3}\right) \mathrm{A}$.

This estimate is now compared to theoretical estimates. Estimates are based on the 'hot-tail' mechanism, whereby as pictured in Fig. 2(a) a residual population of higher energy electrons from the pre-disruption Maxwellian distribution are accelerated by the electric field to become REs before they can thermalize with the rapidly cooling bulk. The hot-tail mechanism is expected to be dominant in ITER disruptions and is also computed to be largest for these DIII-D experiments, overwhelming the alternate Dreicer mechanism [20, 17]. Further, no pre-disruption RE populations are expected in these conditions. A first comparison is given to the original hot-tail theory of Ref. [18, where the RE seed population is calculated in the limit that the rate of bulk $T_{e}$ cooling is much faster than the electron equilibration time, essentially reducing the problem to counting the number of electrons above a certain energy in the pre-disruption Maxwellian (red region in Fig. 2[a]). This calculation results in a seed estimate that is several orders of magnitude too large, and indeed the observed TQ duration (0.3 $\mathrm{ms}$ ) is actually comparable to the calculated electron thermalization time (0.1-0.3 ms). This RE seed estimate is unphysical, as it exceeds the pre-disruption current. This is possible in this model as the RE seed is assumed to be accelerated to relativistic energy, whereas in reality the finite available flux would limit the RE energy such that the RE seed current would equal the pre-disruption current. A second comparison is to the more recent hot-tail theory of Ref. 19. This theory includes a self-consistent prediction for the electric field evolution by considering the plasma power balance during the TQ, assuming the line-radiation as a dominant energy loss mechanism, and including RE generation. This is important because the generation of REs can slow the TQ, which in turn reduces the inductive electric field generation, which in turn reduces the RE generation rate. A limitation of the present model, however, is that the Ar impurity is assumed to be deposited uniformly and instantaneously across the profile, whereas a realistic pellet presents a localized and gradually evolving impurity source. In this model, a bulk cooling rate of $0.5-2.0 \mathrm{~ms}$ is predicted, and the predicted RE seed is now significantly below measurement. Due to the exponential sensitivity of the hot-tail mechanism, factors of two in bulk cooling rate equate to orders of magnitude different RE seed currents. Further details of the predicted seed current parametric dependence are shown in Fig. 2(c), where a characteristic non-monotonic dependence of the seed production on $T_{e}$ is found for DIII-D conditions. Taking the experimentally deposited Ar density $\left(n_{A r}\right)$, the model predicts a low seed fraction, though merely doubling the deposited $n_{A r}$ would considerably improve the agreement.

These theories, while both not agreeing with experiment, demonstrate that timedependent treatment of the pellet dynamics together with a self-consistent treatment of 


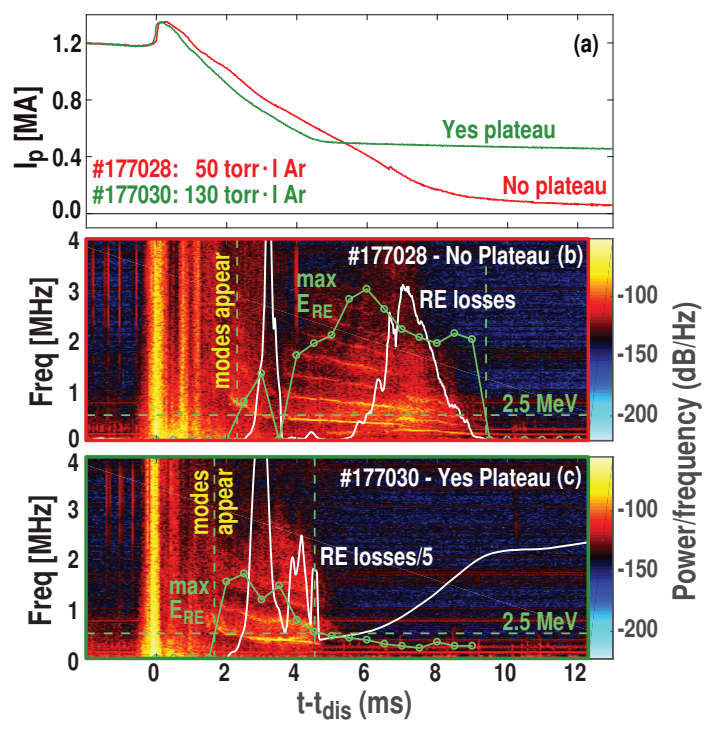

Figure 3. (a) Time evolution of plasma current $\left(I_{P}\right)$ for a discharge that does (green) and does not (red) form a mature RE plateau. (b) The discharge that does not form the plateau exhibits intense and long-lasting instability activity in the $\mathrm{MHz}$ range, while (c) the discharge that does form a RE plateau has shorter and less intense mode activity. Modes appear above a critical RE energy $\left(E_{R E}\right)$ (green) of $2.5 \mathrm{MeV}$, and are correlated with RE loss (white) as measured by a distant HXR detector [22].

the RE generation and bulk cooling rate are essential to accurately predict the RE seed generation rate. In particular, recent theory finds that a significant fraction of the preTQ electron energy can be transfered to ions during the pellet cloud expansion, affecting the power balance during the TQ [21]. Furthermore, measurements of the bulk cooling rate are themselves of great value in constraining $\mathrm{RE}$ seed generation models. Small differences in the predicted bulk cooling rate can give rise to orders of magnitude different RE seed currents. Future measurement work in this area would benefit from routine diagnosis of the bulk cooling rate through improvements to the Thomson Scattering diagnostic time-resolution, as in most cases ECE-based $T_{e}$ is unavailable due to cut-off.

\subsection{Kinetic Instability and RE Seed Survival}

The observed kA-level RE seeds must undergo avalanche multiplication in order to form the 100s kA-level RE plateau. This sensitive time presents another opportunity to avoid the RE plateau phase. On DIII-D, it is found that RE plateau formation is unreliable, as tabulated in Fig. 2 of Ref. [23]. Often times, the RE seed does not survive into the plateau phase. Previous experimental and theoretical work proposed a role for MHD activity in preventing the RE seed amplification [24, 9] and aimed to explain RE formation dependencies on plasma shape and magnetic field.

Recent studies propose a novel candidate mechanism whereby kinetic instabilities excited by the RE beam can lead to enhanced RE spatial transport that can inhibit RE seed survival into the plateau [22]. This work employs a unique combination of high- 

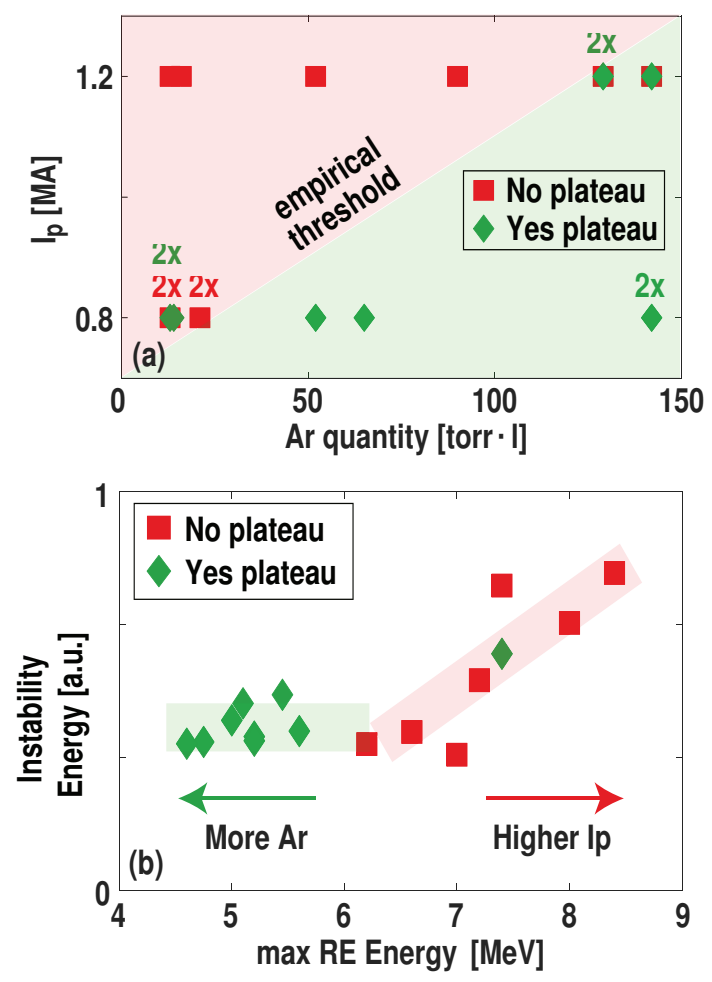

Figure 4. (a) Empirical thresholds in $I_{P}$ and primary injection Ar quantity, where the pre-disruption plasma inventory is $\approx 8$ torr-l ( 1 torr- $1=3.21 \times 10^{19}$ atoms). (b) Thresholds are correlated with the RE-driven instability energy (defined as the integral of the $|\delta B|^{2}$ spectrum from 0.1 to $5 \mathrm{MHz}$ and over the duration of the instability) as well as the maximum $E_{R E}$ (measuring over the instability duration).

frequency magnetic probes [25] to observe the kinetic instability and hard X-ray (HXR) spectrometers [26] to diagnose the RE properties that excite instabilities. As shown in Fig. 3, MHz-frequency magnetic fluctuations are observed and found to be particularly intense and long-lived in discharges that fail to form RE plateaus. These fluctuations are in turn found to be excited when the maximum RE energy $\left(E_{R E}\right)$ exceeds a threshold $2.5 \mathrm{MeV}$, as measured by HXR spectrometry. Looking with high time resolution, it can be seen that spikes in distant HXR detectors (indicating RE loss) are preceded by periods of intense fluctuation activity. This indicates the fluctuations are driving at least some RE loss. While the mode can be said to drive some RE loss, whether or not the mode-driven RE loss is solely responsible for preventing plateau formation is still under study (ie, whether there is correlation but not full causation).

Correlations supporting the mode-driven loss hypothesis have been identified. Variations of the primary injection Ar massive gas injection (MGI) quantity and predisruption $I_{P}$ are carried out, and find threshold-like behavior for RE plateau formation. As shown in Fig. 4(a), more Ar is necessary to form the RE plateau at high $I_{P}$. This is a counter-intuitive result as higher $I_{P}$ should facilitate RE formation and thus reduce the external Ar needed. It should be noted that here $B_{T}$ was unchanged and thus the edge safety factor $\left(q_{95}\right)$ decreases from 4.5 to 3.1 , leaving open the possibility that different 


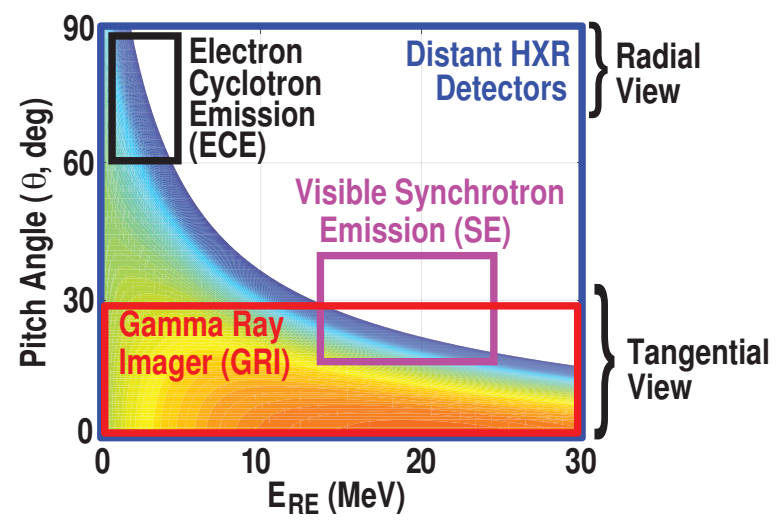

Figure 5. Diagnostics used to infer the RE distribution function (color contours) along with their rough area of sensitivity in the RE phase space. Note the pitch angle is primary resolved by changing the viewing angle towards the RE beam.

MHD behavior may impact this result. HXR spectrometry finds that decreasing the Ar quantity and increasing $I_{P}$ both increase the maximum $E_{R E}$, likely due to an decrease in collisional damping and an increase in available flux, respectively. As shown in Fig. $4(\mathrm{~b})$, this increase in $\max E_{R E}$ is found to correlate with an increase in kinetic instability energy (calculated by integrating the magnetic spectrogram over frequency and time) as well as with inhibited RE plateau formation. In cases with the same pre-disruption $I_{P}$, the differentiation in $E_{R E}$ is found before $I_{P}$ differentiates, indicating that differences in the available flux are not to blame (at fixed $I_{P}$ ). These observations supporting an important role for the kinetic instability in these conditions.

Preliminary modeling indicates that the observed mode is a compressional Alfven eigenmode (CAE) found at the low frequency extreme of the fast wave dispersion relation [27], and relies on enhanced pitch-angle scattering [28, 29] as well as non-monotonicity in the RE distribution function for its excitation. Future work in this area will aim to better diagnose the kinetic instability mode structure with newly installed toroidal arrays of high-frequency magnetic probes, and begin to assess the possibility of active launch of these instabilities through low-power antenna loading studies. As will be discussed in Sec. 6.1, the observation of naturally excited MHz-range kinetic instabilities in this sensitive phase of the RE formation opens novel opportunities to avoid the RE plateau.

\section{Quiescent Scenarios: RE Mitigation in Controlled Conditions}

Attention now turns to understanding the dissipation of the RE plateau, termed RE mitigation. As the plateau stage is quasi-stationary over $\approx 100 \mathrm{~ms}$, ample time exists to apply secondary mitigating actions to dissipate the plateau prior to the first-wall strike. Recent DIII-D experiments conducted in 'quiescent' scenarios are reviewed. Quiescent scenarios use extremely low density Ohmic flat-top plasmas to slowly excite REs through the Dreicer mechanism [20]. These Dreicer REs are then dissipated (or 
not) by varying the background plasma density, magnetic field, impurity content, or combinations thereof [30]. These scenarios offer advantages in plasma profile control, measurement, and model validation. For example, all of the inputs necessary to compute the RE distribution function $\left(f_{e}\right)$ are easily provided. The RE distribution function itself is measured in these plasmas by a combination of diagnostics, each sensitive to different parts of the RE phase space, as shown in Fig. 5. This section will describe the application of each of these diagnostics to measure aspects of $f_{e}$ and its dependence on plasma parameters.

Experiments in the quiescent regime find: 1) $\mathrm{RE} f_{e}$ measured via bremsstrahlung agree with 0-D (spatial) and 2-V (phase space) Fokker-Planck (0-D 2-V FP) modeling in terms of the basic $f_{e}$ shape and parametric dependencies on normalized collisionality and synchrotron damping. 2) Synthetic diagnostics of synchrotron images have been newly developed and support bremsstrahlung measurements, enabling improved resolution of $f_{e}$ and especially its pitch-angle distribution. 3) 100-200 MHz-frequency RE-driven kinetic instabilities are observed for the first time, and when the effect of these and other instabilities are included in a quasi-linear diffusion framework previously reported RE dissipation anomalies are captured. 4) Inclusion of kinetic instabilities also resolves previously discrepant electron cyclotron emission (ECE) spectral measurements.

\subsection{RE Distribution Measurement via Bremsstrahlung}

Energy-resolved RE measurements are made with a unique tangentially viewing pinhole camera (termed the Gamma Ray Imager, GRI) equipped with HXR spectrometers viewing a subset of available pixels [31, 26]. HXRs are emitted by the RE population via bremsstrahlung when they undergo Coulomb collisions with the background plasma ions, and the HXR energy is measured via a scintillation pulse when the HXR interacts with a crystal within each spectrometer. Collecting discrete HXR pulses over a suitably long time interval generates a statistical HXR energy spectrum $\left(f_{\gamma}\right)$, and knowledge of the expected $f_{\gamma}$ from mono-energetic RE beams enables inversion of the $f_{\gamma}$ to the RE distribution function $\left(f_{e}\right)$ [32, 26, 33, 34]. First experiments focused on varying background plasma parameters such as the electron density $\left(n_{e}\right)$, electric field $\left(E_{\phi}\right)$, $Z$, and toroidal field $\left(B_{T}\right)$ to isolate the dependence of the RE distribution function $\left(f_{e}\right)$ on collisional damping (scales like $E_{\phi} / n_{e}$ ) and synchrotron damping (scales like $\left.B_{T}^{2} / n_{e}\right)$. Measurement of all important parameters in time enables computation of the model-predicted $f_{e}$ in time by solving the 0-D 2-V FP equation [35, 13]. Rigorous comparisons with experiment are possible by comparing the model-predicted $f_{e}$ with experiment over all time in a discharge, though to improve counting statistics, pulses are counted over 1-2 s periods with stationary background plasma conditions (but not necessarily stationary $f_{e}$ ) and compared to the model-predicted $f_{e}$ averaged over the same period. To isolate $f_{e}$ effects, care is taken to have similar RE growth phases (at the same plasma parameters), prior to modifying the background plasma parameters.

Comparing the $f_{e}$ obtained by experiment and modeling in Fig. 6, qualitative trends 

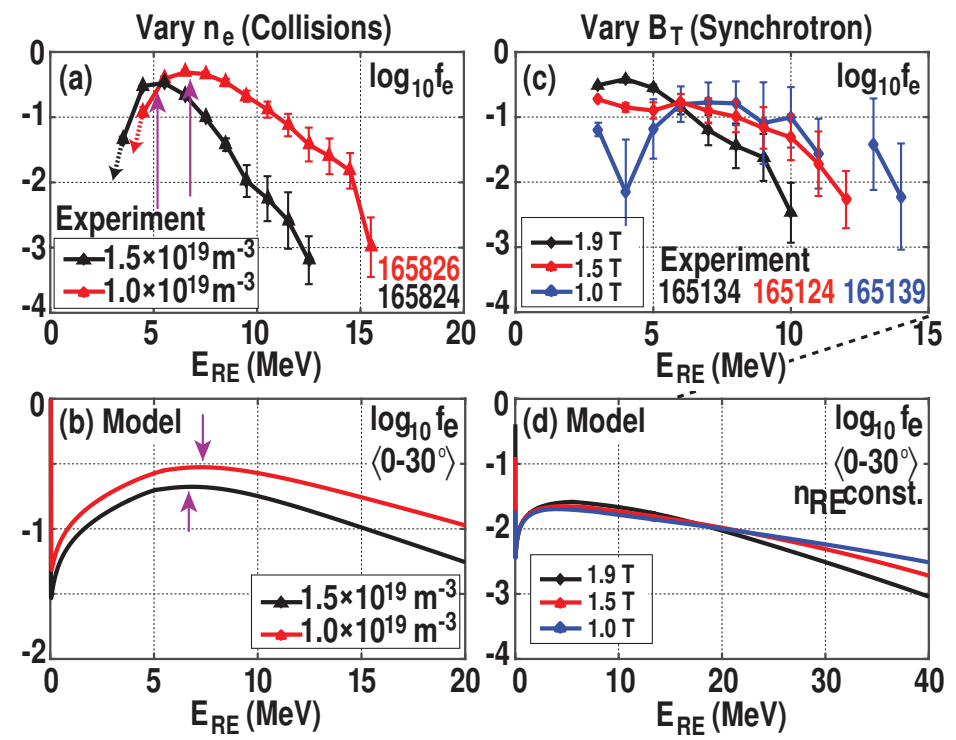

Figure 6. Variation of $f_{e}$ as (a,b) collisional and (c,d) synchrotron damping is varied. $(\mathrm{a}, \mathrm{c})$ Experimental $f_{e}$ are obtained by inverting $f_{\gamma}$ assuming spatial $f_{e}$ homogeneity. $(\mathrm{b}, \mathrm{d})$ 0-D 2-V FP modeling computes the $f_{e}$ evolution over the entire discharge. Time intervals with stationary background plasma conditions are compared. The modeled 2$\mathrm{D} f_{e}$ is integrated from pitch angles of 0 to 30 , matching the experimental view. Purple arrows in $(\mathrm{a}, \mathrm{b})$ highlight the peak of $f_{e}$. Experimental error bars are propagated from underlying $f_{\gamma}$ counting statistics. Note different axis limits are used. Reprinted with permission from Ref. [33.

with $n_{e}$ and $B_{T}$ are captured and some discrepancies identified [33, 36]. Non-monotonic $f_{e}$ features are found at the $E_{R E}$ predicted by time-dependent 0-D 2-V FP modeling [see purple arrows in Fig. 6(a,b)]. These features are long-lived and formed by the interplay of acceleration via $E_{\phi}$, drag via electron-electron collisions, pitch-angle scattering via electron-ion collisions, and energy loss via synchrotron radiation [13, 37]. While nonmonotonic features in the $f_{e}$ may appear only a curiosity, their presence is important because they can provide a source of free energy for kinetic instabilities driven by the RE beam, as will be described in Sec. 3.3. Considering the importance of background plasma parameters, increasing collisional damping by varying $n_{e}$ shifts the full $f_{e}$ to lower energy (Fig. 6[a,b]), by an amount consistent with the theory prediction. In contrast, increasing synchrotron damping by varying $B_{T}$ shifts the high- $E_{R E} f_{e}$ towards lower energy (Fig. 6]c,d]), though quantitatively observed $B_{T}$ effects are larger than predicted. The under-prediction of $B_{T}$ effects may be due to the neglect of spatial gradients in the 0-D 2-V FP modeling, as the next section will show the synchrotron emission is preferentially found inboard of the magnetic axis (where $B_{T}$ is considerably larger). Additionally, a recent full-orbit treatment of RE dynamics finds the synchrotron power substantially increases as compared to the guiding center approximations used in 0-D 2-V FP modeling [38].

While the basic structure and parametric dependencies are predicted, the behavior 
at high energy is anomalous, with the experimental $f_{e}$ falling off much more sharply with energy than the modeled $f_{e}$. A candidate resolution to this discrepancy will be provided in Sec. 3.3. In the time domain, increasing $B_{T}$ increases the decay rate at high $E_{R E}$ while increasing $n_{e}$ increases the RE decay rate at all $E_{R E}$.

Considering information in the spatial domain, energy-dependent radial gradients of the RE population are observed, enabling future model validation efforts to specifically target RE spatial transport effects due to either MHD instabilities [39, 40, 16, 41], imposed 3-D fields [42, 43], or inherent processes [44]. Furthermore, simultaneously viewing the same flux surface with different sight-line angles to the magnetic field enables deconvolution of pitch-angle information. Future work is planned to use the full spatial resolution of the GRI diagnostic combined with orbit tomography techniques [45] to enable higher dimensionality inversions of $f_{e}$, such as both energy and pitch-angle.

It is also important to point out that in this first deployment of the GRI, the system was characterized by a limit in maximum allowable HXR flux of about $10^{4}$ counts/s per detector, due to the $\mu$ s-scale scintillation pulse time of the selected BGO crystal. This flux limit was compatible with quiescent scenario RE beams, but not the more intense post-disruption beams. The more recent work summarized in Sec. 2.2 utilized a new scintillation crystal (LYSO), with ns-scale scintillation pulses enabling $10^{7}$ counts/s per detector [46, 47]. Future GRI development work is focused on increasing the number of these more advanced detectors deployed on the GRI to better diagnose post-disruption conditions.

\subsection{RE Distribution Validation via Synchrotron Imaging}

Visible and infrared images of the $\mathrm{RE}$ beam are ubiquitous in the worldwide RE experimental program (see Table 1 in Ref. [51]). These images are generally dominated by synchrotron emission from the eV-level photons emitted by REs following curved trajectories in real space due to gyromotion and the curvature of the toroidal magnetic fields. Only very recently have synthetic diagnostics for this emission been sufficiently developed to enable model-experiment validation of the RE $f_{e}$ and spatial geometry [48, 52, 53, 38, 49].

As seen in Fig. 7, these tools are now able to reproduce experimental synchrotron images with appropriate choice of inputs. These studies also confirm the accessed region of RE phase space shown in Fig. 5, indicating particles must have both fairly high energy $(\gtrsim 10 \mathrm{MeV})$ and high pitch angle $\left(\gtrsim 15^{\circ}\right)$ to be detected in synchrotron images. Synchrotron emission images are thus particularly useful to extract information about the pitch-angle distribution of the high energy REs as well as their spatial extent. Spatial sensitivity arises from camera technology and focusing optics, which generally yield images of very high resolution $(\approx 100 \mathrm{~s} \times 100$ s of pixels, as compared to $\approx 10 \times 10$ maximum pixels in the GRI).

Surprisingly, it is found in Ref. [52] that the synchrotron image computed for a $f_{e}$ obtained by $0-\mathrm{D} 2-\mathrm{V}$ FP simulations could not match the experimental measurements, 

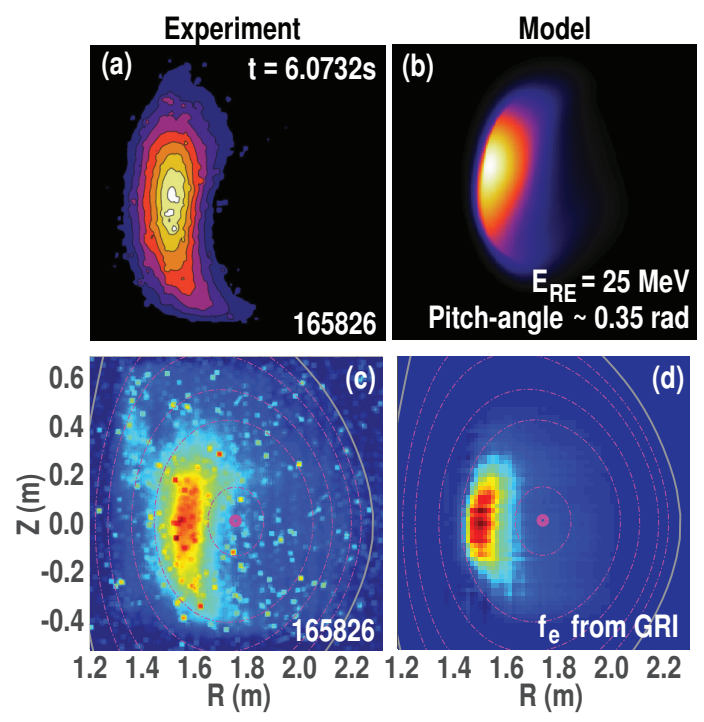

Figure 7. Comparison of synchrotron camera (a,c) experimental images at $890 \mathrm{~nm}$ to $(\mathrm{b}, \mathrm{d})$ synthetic diagnostics using two $f_{e}$ assumptions. The model of (b) uses a best-fit radial distribution, mono-energetic $E_{R E}$, and pitch-angles centered around $0.35 \mathrm{rad}$ [48], while the model of (d) uses a best-fit radial and pitch-angle distribution with the bremsstrahlung-inferred $f_{e}$ [49, 50].

regardless of spatial distribution selected. Instead, an ad-hoc increase of the pitch-angle and decrease of $E_{R E}$ is required to obtain the good match shown in Fig. 7(b). This provides additional evidence that the $f_{e}$ fall-off at high energy is inaccurately predicted by $0-\mathrm{D} 2-\mathrm{V}$ FP simulations.

Complementary studies described in Ref. [49] took an alternate approach to match the experiment. Instead of computing $f_{e}$ from $0-\mathrm{D} 2-\mathrm{V}$ FP simulations, the experimental $f_{e}$ 1-D energy spectrum (from bremsstrahlung) was input and the pitch-angle and spatial distribution scanned until the model best matched the experimental synchrotron image. This approach also reveals that the 0-D 2-V FP computed pitch angle distribution is inaccurate, and identified either spatially inhomogeneous magnetic fields or full orbit effects as modifying the pitch angle distribution in a way that brought the computed image to agreement with the experimental image, as shown in Figs. 7)(c,d).

Both studies thus demonstrate the inadequacy of the 0-D 2-V FP treatment at high energy and high pitch angle (where the synchrotron image originates), and opens the door to new physics being important to capture the $f_{e}$ in this region of phase space. Future work in this area will aim to extend model validation of synchrotron images to fully 3-D magnetic configurations (ie, tokamak discharges with magnetic islands) [50], and incorporate synchrotron image data in unified multi-diagnostic inversions to better infer $f_{e}$ [45]. 


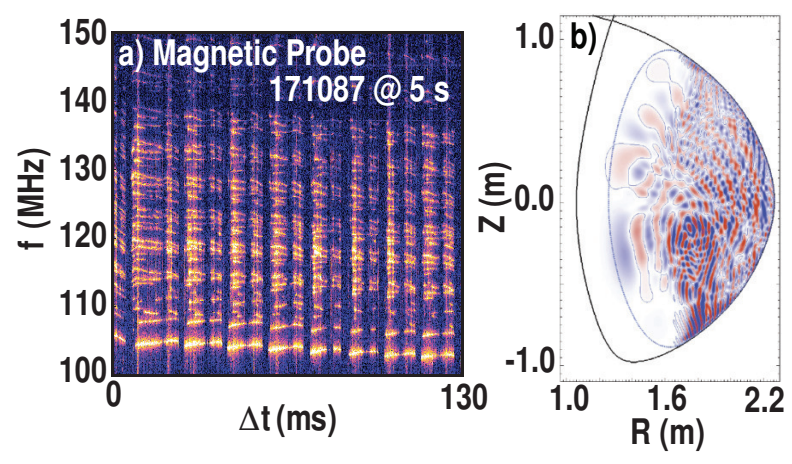

Figure 8. (a) Measurements from magnetic probes reveal high-frequency RE-driven kinetic instabilities in quiescent scenarios, with a characteristic banded structure and intermittency. $\quad B_{T}$ is decreasing during the plotted interval alongside the observed mode frequencies. (b) AORSA predictions of the kinetic instability perturbed electric field mode structure at $140 \mathrm{MHz}$ [54.

\subsection{RE Dissipation via Kinetic Instabilities}

Previous sections have highlighted areas of $f_{e}$ agreement and disagreement with 0-D 2-V FP modeling. However, the most glaring discrepancy with 0-D 2-V FP modeling concerned the anomalous decay of RE emission at low levels of normalized collisionality. Normalized collisionality is given by the ratio of the toroidal electric field $\left(E_{\phi}\right)$ to the 'critical' electric field $\left(E_{\text {crit }}\right)$, as first presented in Ref. [55]. $E_{\text {crit }}$ is the electric field whose acceleration force balances collisional drag forces at fully relativistic energies, and is proportional to $n_{e}$. Multi-machine quiescent regime experiments had previously found RE emission decay with $E_{\phi} / E_{c r i t}$ values of 5 or more, indicating the effective $E_{c r i t}$ was significantly underpredicted [56, 30]. Cast in terms of an effective critical $n_{e}$ for $\mathrm{RE}$ decay, these results indicated the necessary $n_{e}$ was significantly over-predicted. At the time, the most complete $0-\mathrm{D}$ 2-V FP treatments including additional effects such as synchrotron damping and pitch angle scattering predicted $E_{\phi} / E_{c r i t}$ values of just under 2 being sufficient for RE decay in these conditions [13, 57]. Later energy-resolved HXR growth and decay measurements further clarified this picture by finding the strongest anomalous dissipation to low energy [36, 33]. These measurements were hypothesized to be explained by either spatial transport of REs, or by modification of the RE $f_{e}$ and dissipation rate by kinetic instabilities. The isolation of the anomalous loss to low energy is difficult to explain with spatial transport arguments however, since these losses should increase with energy. In contrast, the kinetic instability hypothesis was supported by the observation of non-monotonic features in $f_{e}$, which should be a source of free energy for these instabilities.

In the subsequent DIII-D experimental campaign, dedicated experiments were conducted to measure kinetic instabilities in quiescent plasmas. As shown in Fig. 8 , these experiments successfully observed intense and banded fluctuations in the 100$200 \mathrm{MHz}$ range using high frequency magnetic probes [54]. Variations of $n_{e}$ and $B_{T}$ 

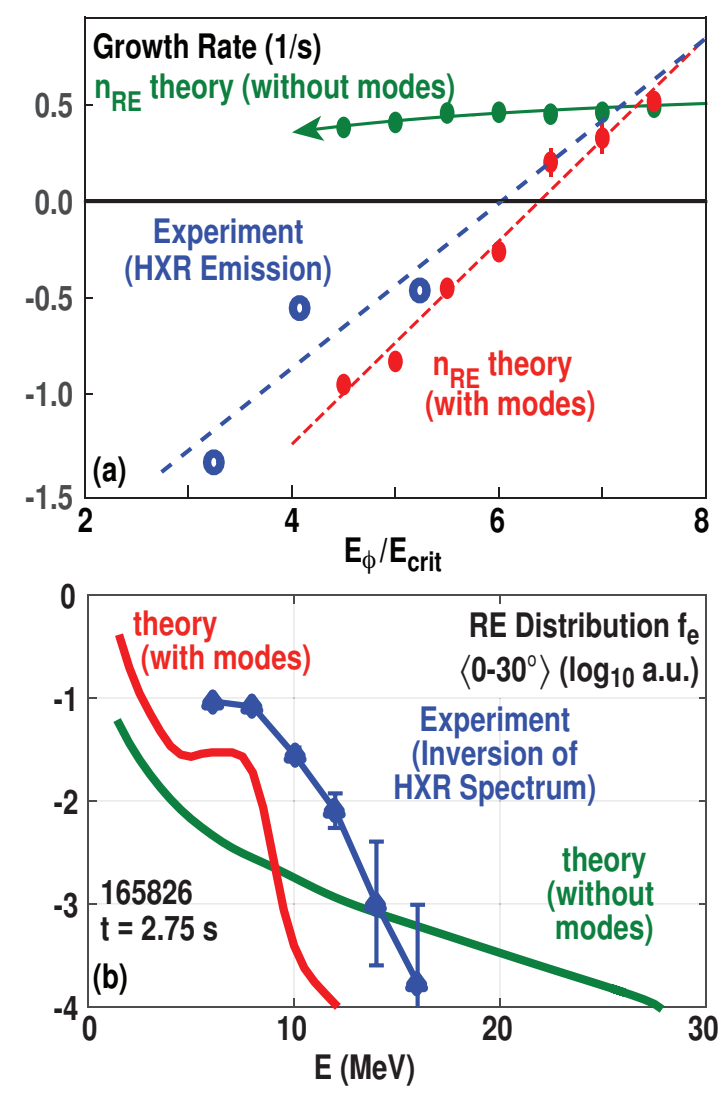

Figure 9. Model-experiment discrepancies in (a) RE growth rate as a function of $E_{\phi} / E_{\text {crit }}$ and (b) RE distribution function are substantially reduced when taking kinetic instability effects into account self-consistently in $f_{e}$ modeling using quasi-linear diffusion 60.

enabled identification of these modes as following the whistler branch of the cold plasma dispersion relation, with wavenumber $k \approx 150 \mathrm{~m}^{-1}$ and small parallel wavenumber $\left(k_{\|} \ll k\right)[58$ ]. Full-wave calculations (using the AORSA code [59]) of the mode structure at this frequency and wavenumber, shown in Fig. 8(b), find a very detailed spatial structure that cannot easily be measured with line-integrated measurements.

In parallel, improvements to the 0-D 2-V FP model were developed to selfconsistently treat the excitation of RE-driven waves from a given $f_{e}$ that then back-react to modify the $f_{e}$ through quasilinear diffusion of the REs in phase space [60]. Building on the framework developed in Ref. [61], this model predicted the existence of the 100$200 \mathrm{MHz}$ waves, and found the dominant drive to be non-monotonicity in energy (as opposed to pitch-angle gradients). This model also predicts a far wider spectrum of modes in the $\mathrm{GHz}$ range, though these modes do not propagate outside of the plasma and thus challenge measurement. The low frequency branch (100-200 MHz) is indeed only detectable because the small gap between the plasma separatrix and the magnetic probe is smaller then the decay length of the evanescent wave in vacuum. It remains an open question whether the predicted GHz frequency instabilities are detectable. 

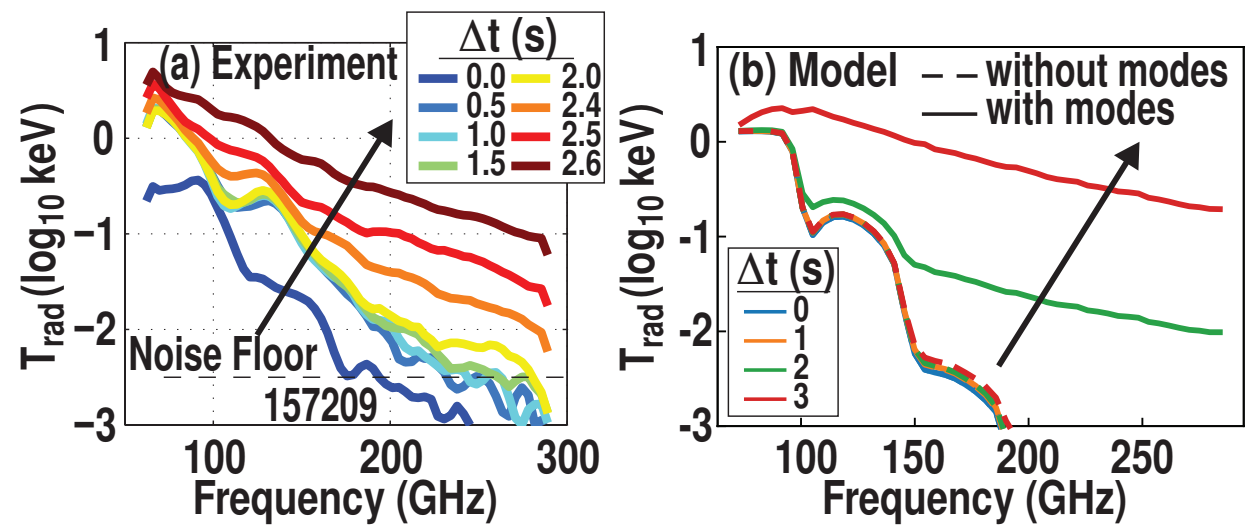

Figure 10. (a) Experimentally observed ECE spectrum evolution and (b) modelpredicted evolution with (solid) and without (dashed) taking kinetic instability effects into account self-consistently in $f_{e}$ modeling using quasi-linear diffusion [62].

The quasilinear diffusion model of Ref. [60] recovers several previously anomalous results. First, as shown in Fig. 9(a), this model predicts a substantial increase in the effective $E_{\phi} / E_{\text {crit }}$ for RE decay due to the RE-driven wave, bringing modeling and experiment largely into agreement on the effective $E_{\phi} / E_{\text {crit }}$. This occurs through a mix of direct wave-energy transfer and enhanced pitch angle scattering that together with synchrotron damping reduces the energy of the REs until they return to the bulk population. Note that while the RE decay rate is energy dependent [36], here the comparison is of aggregate quantities (the distant HXR detector in experiment, and the total RE density in modeling). Considering the energy dependence, the discrepancies between the measured and modeled fall-off of the RE $f_{e}$ at high energy are decreased when the kinetic instabilities are included, as shown in Fig. 9(b). It is interesting to note that in the Ref. [60] model, it is the undetected GHz-level modes that provide the bulk of the dissipation, as opposed to the observed 100-200 MHz modes.

\subsection{Consistency with Electron Cyclotron Emission Spectra}

Final supporting evidence for the importance of RE-driven kinetic instabilities can be found by consideration of the electron cyclotron emission (ECE) spectrum. As shown in Fig. 5. this diagnostic is sensitive to very high pitch angle (due to the radial view) and few $\mathrm{MeV} E_{R E}$. Previous work in DIII-D had identified an unusually abrupt rise in the ECE emission and spectral hardness as the quiescent scenario RE beam matured late in time [63]. This is shown for a representative DIII-D discharge in Fig. 10(a). Using the quasilinear diffusion model, a similar effect is found, as shown in Fig. 10(b) 62]. The rapid rise in ECE can be interpreted as the indirect effect of the onset of intense REdriven wave activity, as these waves scatter REs into the perpendicular direction and thus increase the ECE emission. Later studies reported in Ref. [58] find a supporting correlation between ECE intensity and the presence of 100-200 MHz RE-driven modes. 


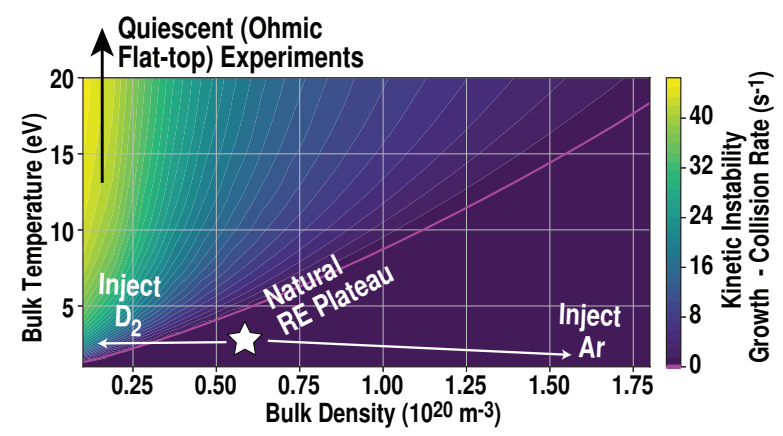

Figure 11. Expected normalized growth rate of high frequency ( $\approx 1 \mathrm{GHz})$ kinetic instabilities in DIII-D conditions as a function of $T_{e}$ and $n_{e}$, using the instability theory of Ref. 13. Modification of bulk parameters during RE plateau experiments by injection of high- $\mathrm{Z}$ and low- $\mathrm{Z}$ gas is shown.

\section{Post-disruption scenarios: mitigation of the mature RE plateau}

Attention now turns to RE dissipation experiments in mature post-disruption RE beams on DIII-D. First, the advances in the quiescent regime are discussed in terms of their applicability to post-disruption RE plateaus. MHz-frequency kinetic instabilities are observed for the first time in this regime. Second, recent experiments on high-Z impurity injection are discussed, highlighting observed dissipation saturation resulting from saturated assimilation of high-Z impurity into the RE beam.

\subsection{Kinetic instabilities in the RE plateau}

While quiescent regime experiments described in Sec. 3 were partially motivated by their good non-dimensional match to ITER RE plateaus in terms of collisional and synchrotron damping effects, the prospect of kinetic instabilities as the dominant anomalous loss mechanism raises new challenges for ITER-relevance. This is because the quiescent Ohmic plasmas, with keV-level bulk $T_{e}$, are much more likely to excite kinetic instabilities due to their low collisionality (between bulk particles). These collisions are predicted to be the main damping mechanism for the considered instabilities. In contrast, the bulk plasma in RE plateaus will naturally be rather cold, dense, and thus very collisional. Note non-thermal normalized collisionalities are comparable in both regimes, as quantified by $E_{\phi} / E_{\text {crit }}$.

As shown in Fig. 11 (taken from the theory of Ref. [13] but applied to DIII-D), cold and dense conditions are poorly suited to excite kinetic instabilities. This is because the collisional coupling between bulk particles is very strong in this parameter regime. Use of argon (Ar) as a secondary injection actuator further pushes the RE plateau away from the conditions to excite instabilities. For this reason, kinetic instabilities had been assumed to be inaccessible for RE dissipation in ITER RE plateaus, requiring an unachievably high $T_{e}$ [13].

Two recent findings call this assumption into question and potentially open new 


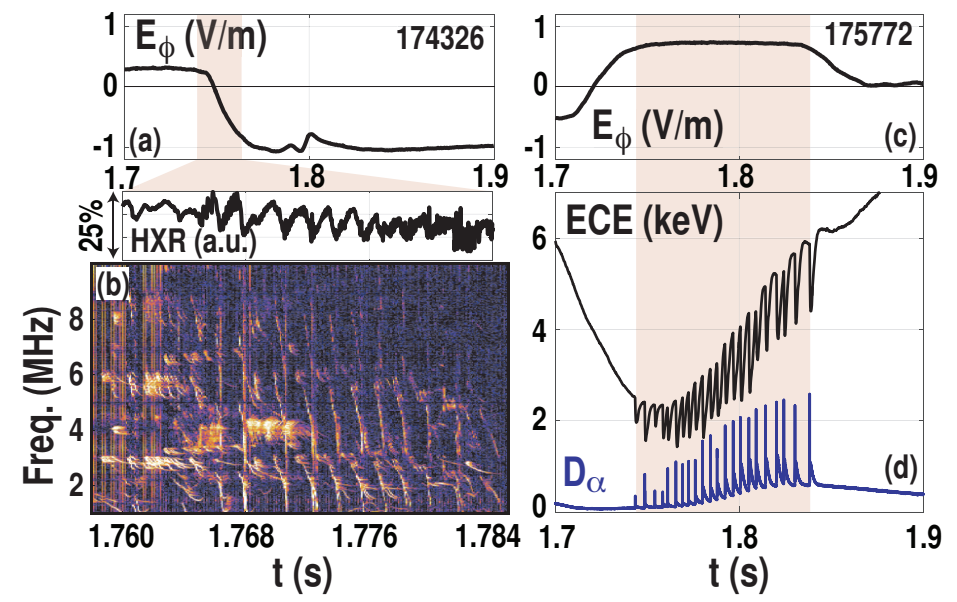

Figure 12. Observation of kinetic instability in DIII-D mature RE plateaus after $\mathrm{D}_{2}$ injection. Instabilities are observed during the shaded time-intervals at very high negative (a,b) and positive (c,d) $E_{\phi}$.

opportunities for RE control. The first is the finding already discussed in Sec. 2.2. whereby MHz-frequency instabilities are observed during the RE formation phase despite the cold and dense (ie, collisional) conditions. Note that the analysis of Fig. 11 cannot at present include instabilities below the ion cyclotron frequency ( $20 \mathrm{MHz}$ in DIII-D). This is due to the global eigenmode nature of the few $\mathrm{MHz}$ instabilities prohibiting a 0-D spatial treatment as done in Ref. [13].

This is a subject of active study, but it may be that the very low frequency of these instabilities (below the ion cyclotron frequency) modify the collisional damping requirements such that the waves can be driven in cold and dense conditions. Furthermore, the bulk collisionality competes against the instability growth rate, which is itself set by the free energy in the $f_{e}$ gradients. Thus, it may be that even in very collisional conditions a sufficiently unstable $f_{e}$ can still be achieved to enable instability growth.

The second finding is the dramatic response of the RE plateau to deuterium $\left(\mathrm{D}_{2}\right)$ injection that demonstrates a path to reducing the bulk collisionality in post-disruption RE plateaus. As first described in Ref. [64, $\mathrm{D}_{2}$ injection is found to rapidly reduce the bulk $n_{e}$ by at 1-2 orders of magnitude, often to measurement zero. The mechanism for this process is not understood. A candidate hypothesis is that the RE distribution function after $\mathrm{D}_{2}$ gas injection is poorly overlapped with the ionization cross section, resulting in recombination and deconfinement of the bulk population. This hypothesis is supported by the response to auxiliary power injection via neutral beam or electron cyclotron heating. Auxiliary heating is found to rapidly increase the bulk $n_{e}$ back to pre- $\mathrm{D}_{2}$ levels. Regardless of mechanism, the achieved low density drastically reduces the collisionality and eases the excitation requirements for RE-driven instabilities.

A preliminary study of wave-excitation in the low $n_{e}$ RE plateaus identified two distinct regimes where RE-driven instability is found, as shown in Fig. 12. Both require 
active modification of the electric field $\left(E_{\phi}\right)$ to observe instability, achieved by ramping the Ohmic solenoid. This indicates that when holding $E_{\phi}$ to maintain constant $I_{R E}$ the accessed $f_{e}$ is stable to any observed instability. At negative $E_{\phi}$ similar frequency instabilities to those of Sec. 2.2 are seen, though now with highly non-linear chirping behavior. The negative $E_{\phi}$ suggests that low RE energies are needed to excite this class of instability. At high $E_{\phi}$, a second class of instability is found. Unlike the first, this class strongly effects the non-thermal ECE emission, giving rise to large crashes that registered in many diagnostics (such as $D_{\alpha}$ ). The nature of this instability is not presently understood, and interestingly it exhibits the opposite ECE temporal characteristic (slow rise, fast fall) to the 'Fast Pitch Angle Scattering' (fan) instability reported elsewhere (fast rise, slow fall) [65, 66, 41]. While these two conditions are unnatural due to the large imposed $E_{\phi}$, they demonstrate that kinetic instabilities can be excited in the RE plateau. Interestingly, it should be noted that the instabilities in Sec. 2.2 also existed during periods of high $E_{\phi}$ (but without $\mathrm{D}_{2}$ injection). Further work is needed to understand how exploit these modes for RE dissipation using ITER-relevant actuators, as will be discussed in Sec. 6.1.

\subsection{High-Z Injection: Impact of RE Plateau Vertical Loss and Dissipation Saturation}

A candidate approach for mitigation of the RE plateau in ITER is the injection of high-Z gas (likely Ar) shortly after the RE plateau is formed. Experiments in DIII-D have addressed the related roles of vertical instability and high-Z dissipation saturation. Unlike in DIII-D, where rapid control responses (enabled by nearby copper coils) maintain RE beam positional control through the disruption [68, the control response in ITER (with distant superconducting coils) is expected to be insufficient to maintain position control. As such, RE beams in ITER are expected to drift upwards during the RE plateau over $\approx 150 \mathrm{~ms}$. This allows for only a finite time for a secondary mitigation actuator to reduce the RE beam current and energy.

DIII-D experiments have simulated this situation by developing vertically elongated RE plateaus, applying a small vertical kick, and then freezing shaping coil currents. This allows the RE beam to drift upwards naturally, as shown in Fig. 13(a-c). Repeating this evolution over many discharges while increasing the Ar secondary injection quantity, it is found that the RE current $\left(I_{R E}\right)$ at the final loss could be reduced, as shown in Fig. 13(d). However, the $I_{R E}$ reduction saturated at a critical Ar quantity, indicating that the amount of Ar assimilated into the beam also saturated. Similar observations

have been reported in other vertically unstable $\mathrm{RE}$ experiments [6, 69, 70]. This effect is under study and is thought to be due to a reduction of neutral diffusion into the beam as the ionization fraction and background temperature decreases. Effectively, the diffusion is found to take longer than the finite time available to act [67].

This study is also motivated by recent ITER simulations using the DINA code [71] that found that increasing the Ar secondary injection quantity increased the vertical loss rate, and thus did not reduce the RE current at the final loss [72]. This effect is not 


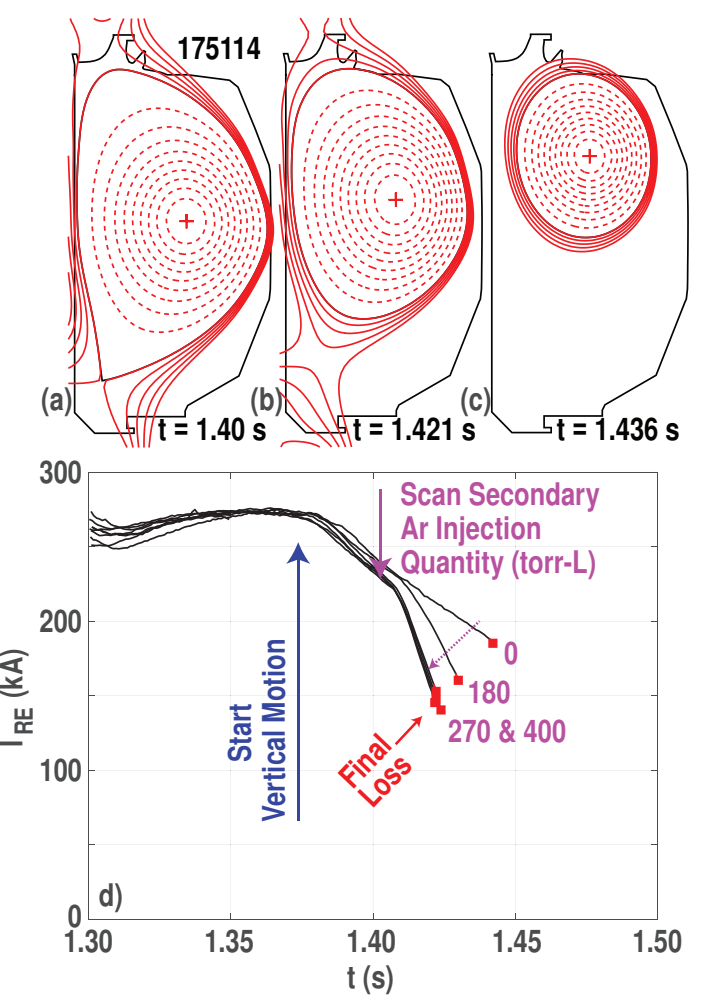

Figure 13. (a)-(c) DIII-D experiments simulate vertically unstable RE beam dynamics. (d) Increasing Ar quantity reduces runaway current $\left(I_{R E}\right)$ at the final loss, until ultimately a seturation of dissipation is observed 67. The pre-injection inventory in the RE beam is about 16 torr-l.

observed on DIII-D. Importantly however, this effect is thought to be due to persistent eddy currents in the ITER vessel that are absent in DIII-D [73, 74]. This is because the ITER vacuum vessel is larger and more conductive than in DIII-D (and indeed all present experiments), as such in ITER the vertical motion is expected to happen faster than the decay of eddy currents in the wall, whereas in DIII-D the eddy currents decay more quickly. Modeling activities are underway to validate analogous simulations with DIII-D data by synthetically modifying the wall conductivity in simulations of both devices.

\section{Final RE Loss to the First-Wall}

While undesirable to encounter, the RE final loss phase still poses important scientific questions to determine the requirements for RE mitigation. Two recent advances are highlighted. 1) A model to predict the Joule heating of the first-wall from a RE strike has been developed and compared to experiment, finding rough agreement as well as a dependence on the $Z$ of the bulk plasma. 2) Evidence is found for MHD kink instabilities triggering complete loss of the RE population when safety factor $\left(q_{a}\right)$ equals 2 , providing an alternate set of final loss phenomenology when MHD instability is encountered. These 


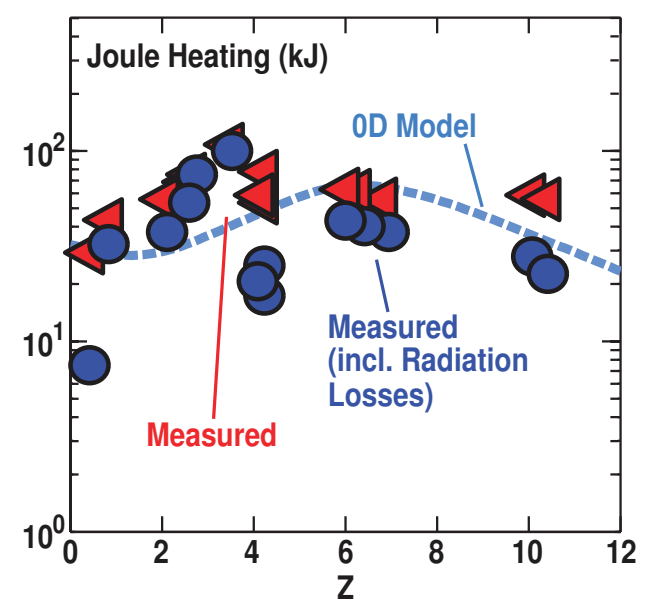

Figure 14. Experimental dependence on bulk plasma $\mathrm{Z}$ of first-wall Joule heating from RE strikes during the final loss and comparison to 0-D lumped circuit parameter model [17.

advances together provide an initial framework for determining when the final loss will begin and for computing its impact.

\subsection{Prediction of Joule Heating}

Prediction of the energy deposited to the first-wall is essential to understand the impact from both mitigated and unmitigated RE strikes, and ultimately to determine the requirements for a successful RE mitigation scheme. New estimates of Joule heating (expected to be the dominant wall heating mechanism in ITER) during the final RE loss have been made and compared to model predictions [17]. Experimental estimates are made by tracking progressive magnetic equilibria using the JFIT code [75] and estimating toroidal loop voltage through changes in magnetic flux. Results are shown in Fig. 14 as a function of the bulk ion effective charge $(Z) . Z$ is varied by changing the secondary injection species (choosing ratios of deuterium, helium, neon, and argon). Measurements are found to vary non-monotonically with $Z$, peaking at an intermediate $Z \approx 6$.

To interpret these measurements, A 0-D circuit model has been developed to predict the Joule heating to the first-wall [76, 77, 17]. The lumped parameter circuit model is used to evolve the total bulk $+\mathrm{RE}$ current and resistance for a given RE loss rate, assumed to be exponentially decaying with a time constant input from experiment. In these cases the loss time is observed to be similar to the avalanche time $(\approx 5 \mathrm{~ms})$. The RE loss time appears to depend on the type of final loss encountered, namely whether the RE beam is MHD stable or not, as will be described in the next section. With these inputs, the model is successful at describing the basic trends in the Joule heating data, being within the experimental scatter for all $Z$. The non-monotonic dependence on $Z$ is understood within the model as a competition between the RE loss time (that increases 

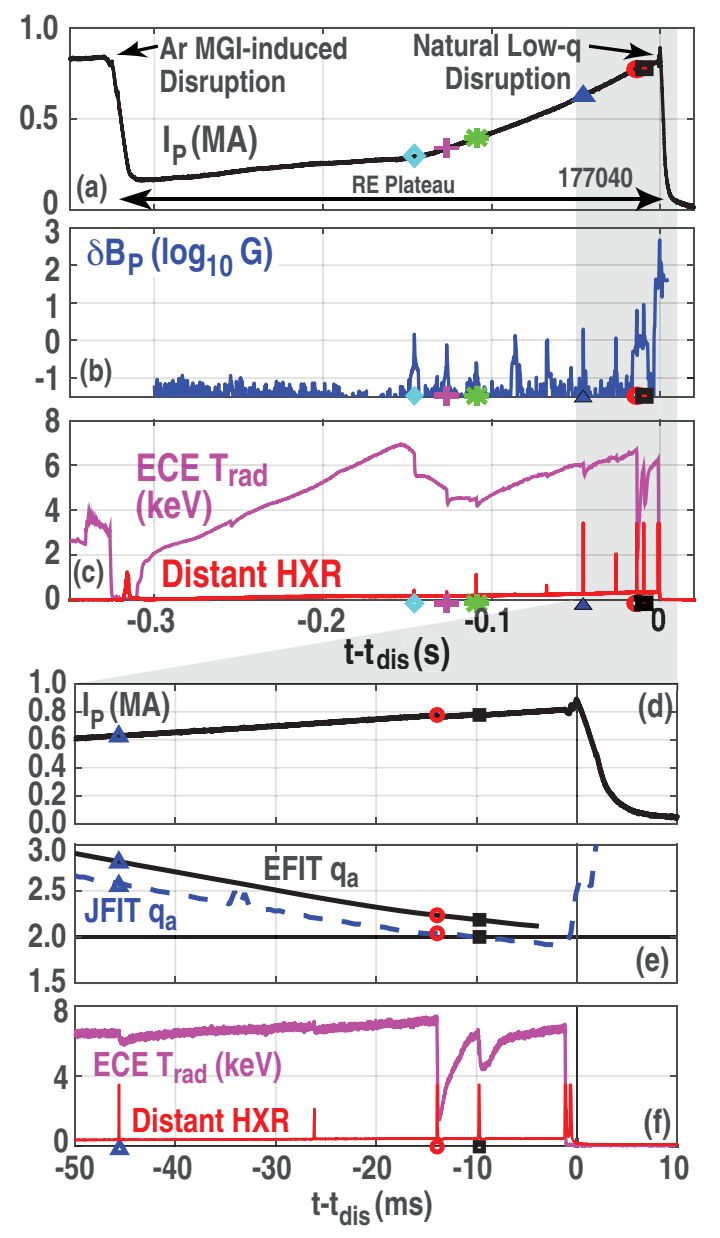

Figure 15. (a) Access to high $I_{P}$ RE beams enables observation of (b) bursty MHD kink modes that drive loss of REs evidenced by (c) ECE and distant HXR measurements. (d-f) These instabilities are severe as $q_{a}$ approaches 2, ultimately leading to loss of the entire RE beam without regeneration.

with $Z$ alongside the avalanche time) and the Ohmic dissipation time (that decreases with Z).

\subsection{Kink Instability at Low Safety Factor}

Recent realization of a nearly MA-level RE plateau on DIII-D enables thorough diagnosis of the phenomenology encountered when approaching the tokamak external kink limit at edge safety factor $\left(q_{a}\right)$ of 2 [78. While difficult to achieve in present experiments, low $q_{a}$ operation is very likely for ITER RE beams owing to the larger RE currents expected in that device [79].

Dynamics are shown in Fig. 15, and are accessed by purposely increasing the RE current to large levels [Fig. 15(a)] while maintaining the RE beam centered in the DIII-D vessel. As RE current in the plateau phase is increased, bursty instabilities are observed in magnetic probe signals [Fig. 15(b)] that cause HXR bursts and drops in internal 
RE emission measured via ECE [Fig. 15(c)]. As $q_{a}$ approaches 2 [Fig. 15(d-f)], the magnetic bursts increase in intensity and eventually lead to a complete loss of the RE beam in dynamics reminiscent of a thermal quench. Comparison of measured magnetic mode structures with MARS-F MHD stability calculations reveals the early modes to be consistent with internal or resistive kinks at higher $q_{a}$ while the later more virulent modes are consistent with internal or external kinks at $q_{a}=2$.

Most importantly, the drive for the RE loss is a global instability with Alfvenic $(\approx 10 \mu \mathrm{s})$ growth rate. This causes a sufficiently complete loss of the RE population that no re-generation is observed, as is expected for more gradual RE loss processes [80]. Both the faster time-scale and the absence of re-generation are not expected in the model described in Sec. 5.1, and its updating to consider the impact of encountering this instability is a subject of future work.

\section{Summary and Discussion}

This work has summarized recent progress in DIII-D experiments aimed at understanding the avoidance and mitigation of REs with application to ITER. These activities have provided novel measurement of the RE population and validation of $\mathrm{RE}$ models, as well as identified areas for future progress.

Considering RE formation, first measurements of the RE seed current demonstrate that present hot-tail theory is not yet able to predict the seed quantity accurately, necessitating improved treatment of the pellet dynamics together with the self-consistent treatment of the bulk $T_{e}$ cooling and RE seed generation. Novel observation of kinetic instabilities in the few $\mathrm{MHz}$ range (likely compressional Alfven eigenmodes) have been made in the RE formation phase, and the intensity of these modes have been correlated with previously unexplained empirical thresholds for RE generation.

Quiescent regime experiments are used to study RE dissipation and validate $\mathrm{RE} f_{e}$ predictions. Agreement is found on the $f_{e}$ parametric dependencies on collisional and synchrotron damping, both in terms of $f_{e}$ shape and dissipation rates. Measurements of bremsstrahlung and synchrotron emission are now used in tandem to resolve energy and pitch-angle effects, respectively. A resolution to long-standing anomalies in the $\mathrm{RE}$ dissipation rate in these experiments is offered by modeling taking into account kinetic instability effects, and novel experiments have observed kinetic instabilities in the 100-200 MHz range with high-frequency magnetic probes.

Post-disruption RE plateau experiments have also observed kinetic instabilities in the mature plateau phase, also in the few $\mathrm{MHz}$ range. Instabilities are observed after the collisional damping rate is reduced through $\mathrm{D}_{2}$ injection. Further experiments studying high-Z collisional dissipation have found that the dissipation rate saturates with high-Z injection quantity, likely due to neutral diffusion rates into the RE plateau being slower than the vertical instability rate in DIII-D.

Considering the final loss phase, a model has been developed that enables estimation of the first-wall Joule heating that is in good agreement with experiment. Additionally, 
controlled access to $q_{a} \approx 2 \mathrm{RE}$ equilibria have identified novel dynamics brought about by large-scale kink instabilities. These dynamics are typified by faster RE loss rates without RE beam regeneration, as expected from large magnitude global 3-D fields deconfining the entire RE population.

\subsection{General Prospects for Kinetic Instabilities}

Recent work has observed RE-driven kinetic instabilities across a wide range of DIII-D RE beam conditions. Generically, quiescent regime instabilities are found at 100-200 $\mathrm{MHz}$, while post-disruption regime instabilities are found at a few MHz. It is remarkable that these instabilities are found in post-disruption conditions, since the bulk $T_{e}$ is only $1-2 \mathrm{eV}$ as measured by Thomson Scattering, and previous models predicted robust collisional suppression of instabilities at this $T_{e}$. These observations have motivated a new interest in the application of kinetic instabilities for RE control, whose prospects are now briefly summarized.

The most promising results thus far were summarized in Sec. 2.2, where the naturally occurring $\mathrm{MHz}$-frequency instability above a threshold amplitude is correlated with the failure of RE plateau formation. If this is a generic finding, it may be that difficulties in forming RE beams in present experiments are due to instabilities of this sort, as opposed to issues arising from low $I_{P}$ (small amplification) or small radius (easier $\mathrm{RE}$ loss). This would present an entirely different basis for extrapolating RE formation from present experiments to ITER. Further experimental work on existing tokamaks equipped with high frequency ( $>\mathrm{MHz}$ ) diagnostics are required to clarify this, ideally with similar primary mitigation actuators to ITER (such as shattered pellet injection). It should also be pointed out that effectively this picture is similar to observations on other tokamaks, replacing MHz-frequency kinetic instabilities with 100s kHz-level broadband MHD turbulence [9, 81] or kHz MHD activity [16, 41]. Note that the RE transits the tokamak (DIII-D major radius $R$ of $1.6 \mathrm{~m}$ ) at the speed of light $(c)$, giving a transit frequency $(c /(2 \pi R))$ of $30 \mathrm{MHz}$. This provides an approximate boundary between instabilities that act on REs in real space $(<30 \mathrm{MHz})$ and those that act on REs in phase space (> $30 \mathrm{MHz}$ ). Consequently both few $\mathrm{MHz}$ instabilities, 10-100s kHz MHD instabilities, and stationary applied 3-D fields can deconfine REs in effectively the same manner [42, 8, 82, 70, 43, 16, 41].

The second result is the instabilities found in the mature plateau (Sec. 4.1). Here, several aspects require resolution. Thus far, observation in DIII-D away from the formation phase requires a reduction of $n_{e}$ (conditions achieved through $\mathrm{D}_{2}$ injection) and modification of $E_{\phi}$ (achieved through ramping the solenoid). Improved understanding of the $\mathrm{D}_{2}$ effect on the RE beam is needed, as at present it is not known if this effect extrapolates to ITER. Furthermore, the nature of the $f_{e}$ changes achieved by varying $E_{\phi}$ require clarification, as the achieved $f_{e}$ may not be accessible in ITER.

In both cases, additional opportunities for control may exist via the active launch of $\mathrm{MHz}$-frequency waves from purpose-built antennas to amplify the naturally occurring or 
marginally stable kinetic instabilities. This speculative proposal bears some resemblance to active fast-ion instability studies [83], and requires additional modeling to determine its possible effectiveness. Active launch of higher frequency waves has also been proposed in the literature [84]. These waves act through phase space re-arrangement (the same mechanism as predicted for the quiescent regime results), as opposed to direct spatial transport. The applicability of higher frequency waves will be crucially dependent on issues of wave accessibility (due to the difficulty of propagation in vacuum) as well as the magnitude of collisional damping. Interestingly, antenna accessibility issues may be significantly alleviated at a few $\mathrm{MHz}$, opening the possibility to couple power into the distant RE beam.

\section{Acknowledgments}

This work was supported by the US Department of Energy under DESC0016452, DE-SC0016268, DE-AC02-09CH11466, DE-FC02-04ER54698, DE-FG0207ER54917, DE-AC05-00OR22725 and DC-AC02-09Ch11466. DIII-D data shown in this paper can be obtained in digital format by following the links at https://fusion.gat.com/global/D3D_DMP. M.H., O.E., and T.F. acknowledge support from the EUROfusion Consortium under Euratom grant agreement No. 633053. D.dCN and L.C. acknowledge support by the Office of Fusion Energy Sciences of the U.S. Department of Energy at Oak Ridge National Laboratory, managed by UT- Battelle, LLC, for the U.S. Department of Energy under Contract No. DE-AC0500OR22725. This research used resources of the National Energy Research Scientific Computing Center, a DOE Office of Science User Facility supported by the Office of Science of the U.S. Department of Energy under Contract No. DE-AC0205CH11231.

This report was prepared as an account of work sponsored by an agency of the United States Government. Neither the United States Government nor any agency thereof, nor any of their employees, makes any warranty, express or implied, or assumes any legal liability or responsibility for the accuracy, completeness, or usefulness of any information, apparatus, product, or process disclosed, or represents that its use would not infringe privately owned rights. Reference herein to any specific commercial product, process, or service by trade name, trademark, manufacturer, or otherwise, does not necessarily constitute or imply its endorsement, recommendation, or favoring by the United States Government or any agency thereof. The views and opinions of authors expressed herein do not necessarily state or reflect those of the United States Government or any agency thereof.

\section{References}

[1] Hender T C, Wesley J C, Bialek J M, Bondeson A, Boozer A H, Buttery R J, Garofalo A M, Goodman T P, Granetz R S, Gribov Y, Gruber O, Gryaznevich M P, Giruzzi G, Günter S, Hayashi N, Helander P, Hegna C C, Howell D F, Humphreys D A, Huysmans G T A, Hyatt A W, Isayama A, Jardin S C, Kawano Y, Kellman A G, Kessel C E, Koslowski H R, LaHaye 
R J, Lazzaro E, Liu Y Q, Lukash V, Manickam J, Medvedev S, Mertens V, Mirnov S V, Nakamura Y, Navratil G A, Okabayashi M, Ozeki T, Paccagnella R, Pautasso G, Porcelli F, Pustovitov V D, Riccardo V, Sato M, Sauter O, Schaffer M J, Shimada M, Sonato P, Strait E J, Sugihara M, Takechi M, Turnbull A D, Westerhof E, Whyte D G, Yoshino R, Zohm H, Group, the Itpa Mhd D and Magnet 2007 Nuclear Fusion 47 S128-S202 ISSN 0029-5515 URL https://doi.org/10.1088/0029-5515/47/6/S03

[2] Lehnen M, Aleynikova K, Aleynikov P B, Campbell D J, Drewelow P, Eidietis N W, Gasparyan Y, Granetz R S, Gribov Y, Hartmann N, Hollmann E M, Izzo V A, Jachmich S, Kim S H, Kočan M, Koslowski H R, Kovalenko D, Kruezi U, Loarte A, Maruyama S, Matthews G F, Parks P B, Pautasso G, Pitts R A, Reux C, Riccardo V, Roccella R, Snipes J A, Thornton A J and de Vries P C 2015 Journal of Nuclear Materials 463 39-48 ISSN 00223115 URL http://linkinghub.elsevier.com/retrieve/pii/S0022311514007594

[3] Hollmann E M, Aleynikov P B, Fülöp T, Humphreys D A, Izzo V A, Lehnen M, Lukash V E, Papp G, Pautasso G, Saint-Laurent F and Snipes J A 2015 Physics of Plasmas 22021802 ISSN 1070-664X URL http://dx.doi.org/10.1063/1.4901251

[4] Boozer A H 2015 Physics of Plasmas 22032504 ISSN 10897674 URL http://dx.doi.org/10. $1063 / 1.4913582$

[5] Rosenbluth M N and Putvinski S V 1997 Nuclear Fusion 371355 URL https://doi.org/10. 1088/0029-5515/37/10/I03

[6] Reux C, Plyusnin V, Alper B, Alves D, Bazylev B and Belonohy E 2015 Nuclear Fusion 55093013 URL https://doi.org/10.1088/0029-5515/55/9/093013

[7] Papp G, Fülöp T, Fehér T, Vries P C D, Plyusnin V V, Alper B and Efda J E T 2013 Nuclear Fusion 53123017 ISSN 00295515 URL https://doi.org/10.1088/0029-5515/53/12/123017

[8] Gobbin M, Li L, Liu Y, Marrelli L, Nocente M, Papp G, Pautasso G, Piovesan P, Valisa M, Carnevale D, Esposito B, Giacomelli L, Gospodarczyk M, McCarthy P J, Martin P and Suttrop W 2018 Plasma Physics and Controlled Fusion 60014036 URL https://doi.org/10.1088/ 1361-6587/aa90c4

[9] Zeng L, Koslowski H R, Liang Y, Lvovskiy a, Lehnen M, Nicolai D, Pearson J, Rack M, Jaegers H, Finken K H, Wongrach K and Xu Y 2013 Physical Review Letters 110235003 ISSN 0031-9007 URL http://link.aps.org/doi/10.1103/PhysRevLett.110.235003

[10] Sommariva C, Nardon E, Beyer P, Hoelzl M and Huijsmans G T A 2018 Nuclear Fusion 58106022 (Preprint 1805.05655) URL https://doi.org/10.1088/1741-4326/aad47d\{\%\}0A

[11] Fülöp T, Pokol G I, Helander P and Lisak M 2006 Physics of Plasmas 13062506 ISSN 1070664X URL http://dx.doi.org/10.1063/1.2208327

[12] Pokol G I, Fülöp T and Lisak M 2008 Plasma Physics and Controlled Fusion 50045003 ISSN 0741-3335 URL http://stacks . iop.org/0741-3335/50/i=4/a=045003?key=crossref . 308f749a1030ccfa1992a626c9eacc27

[13] Aleynikov P B and Breizman B N 2015 Physical Review Letters 114155001 ISSN 0031-9007 URL https://doi.org/10.1103/PhysRevLett.114.155001

[14] Hollmann E M, Austin M E, Boedo J A, Brooks N H, Commaux N, Eidietis N W, Humphreys D A, Izzo V A, James A N, Jernigan T C, Loarte A, Martin-Solis J R, Moyer R A, Muñoz-Burgos J M, Parks P B, Rudakov D L, Strait E J, Tsui C, Van Zeeland M, Wesley J C and Yu J H 2013 Nuclear Fusion 53083004 ISSN 0029-5515 URL http://stacks.iop.org/0029-5515/53/i= 8/a=083004?key=crossref .57e075cb22a5f9e03d097378d8d1d788

[15] Esposito B, Boncagni L, Buratti P, Carnevale D, Causa F, Gospodarczyk M, Martin-Solis J R, Popovic Z, Agostini M, Apruzzese G, Bin W, Cianfarani C, De Angelis R, Granucci G, Grosso A, Maddaluno G, Marocco D, Piergotti V, Pensa A, Podda S, Pucella G, Ramogida G, Rocchi G, Riva M, Sibio A, Sozzi C, Tilia B, Tudisco O and Valisa M 2017 Plasma Physics and Controlled Fusion 59014044 ISSN 0741-3335 URL http://stacks.iop.org/0741-3335/59/ $i=1 / a=014044$ ? key=crossref .1e1c67020c21c6350db783fcc2bfa7c7

[16] Carnevale D, Ariola M, Artaserse G, Bagnato F, Bin W, Boncagni L, Bolzonella T and Bombarda 
F 2019 Plasma Physics and Controlled Fusion 61014036 URL https://doi.org/10.1088/ 1361-6587/aaef53

[17] Hollmann E M, Commaux N, Eidietis N W, Lasnier C J, Rudakov D L, Shiraki D, Cooper C M, Martin-Solis J R, Parks P B and Paz-Soldan C 2017 Physics of Plasmas 24062505 ISSN 1070664X URL http://dx.doi.org/10.1063/1.4985086

[18] Smith H M and Verwichte E 2008 Physics of Plasmas 15072502 ISSN 1070664X URL http: //link.aip.org/link/PHPAEN/v15/i7/p072502/s1\{\&\}Agg=doi

[19] Aleynikov P B and Breizman B N 2017 Nuclear Fusion 57046009 ISSN 0029-5515 URL https://doi.org/10.1088/1741-4326/aa5895

[20] Dreicer H 1959 Phys. Rev. 115 238-249 URL https://dx.doi.org/10.1103/PhysRev.115.238

[21] Aleynikov P B, Breizman B N, Helander P and Turkin Y 2019 Journal of Plasma Physics 85 905850105 ISSN 0022-3778 URL https://www.cambridge.org/core/product/identifier/ S0022377818001332/type/journal\{_\}article

[22] Lvovskiy A, Paz-Soldan C, Eidietis N W, Dal Molin A, Du X D, Giacomelli L, Herfindal J, Hollmann E M, Martinelli L, Moyer R A, Nocente M, Rigamonti D, Shiraki D, Tardocchi M and Thome K E 2018 Plasma Physics and Controlled Fusion 60124003 URL https: //doi.org/10.1088/1361-6587/aae95a

[23] James A N, Austin M E, Commaux N, Eidietis N W, Evans T E, Hollmann E M, Humphreys D A, Hyatt A W, Izzo V A, Jernigan T C, La Haye R J, Parks P B, Strait E J, Tynan G R, Wesley J C and Yu J H 2012 Nuclear Fusion 5213007 URL https://doi.org/10.1088/0029-5515/ $52 / 1 / 013007$

[24] Izzo V A, Humphreys D A and Kornbluth M 2012 Plasma Physics and Controlled Fusion 5495002 URL http://stacks .iop.org/0741-3335/54/i=9/a=095002

[25] Thome K E, Pace D C, Pinsker R I, Meneghini O, Del Castillo C A and Zhu Y 2018 Review of Scientific Instruments 89 ISSN 10897623

[26] Cooper C M, Pace D C, Paz-Soldan C, Commaux N, Eidietis N W, Hollmann E M and Shiraki D 2016 Review of Scientific Instruments 87 11E602 ISSN 0034-6748 URL http://dx.doi.org/ $10.1063 / 1.4961288$

[27] Liu C, Brennan D P and al E 2019 (in preparation)

[28] Zhogolev V E and Konovalov S V 2014 VANT series Nuclear Fusion 3771 URL https: /doi.org/10.21517/0202-3822-2014-37-3-71-88

[29] Hesslow L, Embréus O, Stahl A, Dubois T C, Papp G, Newton S L and Fülöp T 2017 Physical Review Letters 118 1-5 ISSN 10797114 (Preprint 1705.08638) URL https://doi.org/10. 1103/PhysRevLett.118.255001

[30] Paz-Soldan C, Eidietis N W, Granetz R S, Hollmann E M, Moyer R A, Crocker N A, Wingen A and Zhu Y 2014 Physics of Plasmas 21022514 URL http://dx.doi.org/10.1063/1.4866912

[31] Pace D C, Cooper C M, Taussig D, Eidietis N W, Hollmann E M, Riso V and Van Zeeland M 2016 Review of Scientific Instruments 87043507 URL http://dx.doi.org/10.1063/1.4945566

[32] Shevelev A E, Khilkevitch E M, Kiptily V G, Chugunov I N, Gin D B, Doinikov D N, Naidenov V O, Litvinov A E and Polunovskii I A 2013 Nuclear Fusion 53 123004 ISSN 0029-5515 URL http://stacks.iop.org/0029-5515/53/i=12/a=123004?key= crossref.a4585e5f27da3075de13d5a1433fe538

[33] Paz-Soldan C, Cooper C M, Aleynikov P B, Eidietis N W, Lvovskiy A, Pace D C, Brennan D P, Hollmann E M, Liu C, Moyer R A and Shiraki D 2018 Physics of Plasmas 25056105 ISSN 1070-664X URL https://doi.org/10.1063/1.5024223

[34] Shevelev A E, Khilkevitch E M and Kiptily V G 2018 Nuclear Fusion 58016034 URL http: //iopscience.iop.org/article/10.1088/1741-4326/aa8cea/pdf

[35] Aleynikov P B, Aleynikova K, Breizman B N, Huijsmans G T A, Konovalov S V, Putvinski S V and Zhogolev V E 2014 Kinetic modelling of runaway electrons and their mitigation in ITER Proc. of 25th IAEA Fusion Energy Conf. (St. Petersburg, Russia) pp TH/P3-38 URL http:// www-naweb.iaea.org/napc/physics/FEC/FEC2014/fec2014-preprints/319\{_\}THP338.pdf 
[36] Paz-Soldan C, Cooper C M, Aleynikov P B, Pace D C, Eidietis N W, Brennan D, Granetz R S, Hollmann E M, Liu C, Lvovskiy A, Moyer R A and Shiraki D 2017 Physical Review Letters 118 255002 URL https://doi.org/10.1103/PhysRevLett.118.255002

[37] Decker J, Hirvijoki E, Embréus O, Peysson Y, Stahl A, Pusztai I and Fülöp T 2016 Plasma Physics and Controlled Fusion 58 025016 ISSN 0741-3335 URL https://doi.org/10.1088/ 0741-3335/58/2/025016

[38] Carbajal L and Del-Castillo-Negrete D 2017 Plasma Physics and Controlled Fusion 59 ISSN 13616587 (Preprint 1707.03941) URL https://doi.org/10.1088/1361-6587/aa883e

[39] Boozer A H and Punjabi A 2016 Physics of Plasmas 23102513 ISSN 10897674 URL https: //doi.org/10.1063/1.4966046

[40] Bagnato F, Romano A, Grosso A, Buratti P, Tilia B, Jakubowski L, Rabinski M, Gabellieri L, Zebrowski J, Piergotti V, Pacella D, Rocchi G, Giovenale E, Sibio A and Doria A 2018 Plasma Physics and Controlled Fusion 60115010 ISSN 0741-3335 URL https://doi.org/10.1088/ 1361-6587/aae0b3

[41] Causa F and Buratti P 2019 Nuclear Fusion 59046013 ISSN 0029-5515 URL https://doi.org/ 10.1088/1741-4326/aafe2a\{\%\}0A

[42] Papp G, Drevlak M, Fülöp T, Helander P and Pokol G I 2011 Plasma Physics and Controlled Fusion 5395004 URL https://doi.org/10.1088/0741-3335/53/9/095004

[43] Boozer A H 2019 Plasma Physics and Controlled Fusion 61024002 URL https://doi.org/10. 1088/1361-6587/aaf293

[44] Mcdevitt C J, Guo Z and Tang X z 2019 Plasma Physics and Controlled Fusion 61024004 URL https://doi.org/10.1088/1361-6587/aaf4d1

[45] Stagner L and Heidbrink W W 2017 Physics of Plasmas 24092505 ISSN 10897674 URL https://doi.org/10.1063/1.4990391

[46] Dal Molin A, Martinelli L, Nocente M, Rigamonti D, Abba A, Giacomelli L, Gorini G, Lvovskiy A, Muraro A and Tardocchi M 2018 Review of Scientific Instruments 8910 I134 ISSN 10897623 URL https://doi.org/10.1063/1.5038803

[47] Nocente M, Shevelev A E, Giacomelli L, Pautasso G, Tardocchi M, Gin D, Gobbin M, Gorini G, Fernandes A, Herrmann A, Khilkevitch E, Panontin E, Papp G, Pereira R, Salewski M, Tardini G and Valisa M 2018 Review of Scientific Instruments 89 10I124 ISSN 10897623 URL https://doi.org/10.1063/1.5036658

[48] Hoppe M, Embréus O, Paz-Soldan C, Moyer R A and Fülöp T 2018 Nuclear Fusion 58082001 URL https://doi .org/10.1088/1741-4326/aaae15

[49] Carbajal L, Del-Castillo-Negrete D, Paz-Soldan C, Hollmann E M, Moyer R A and Lasnier C J 2018 Pitch-angle Dynamics and Synchrotron Emission of Runaway Electrons in Quiescent and Disrupted DIII-D Plasmas Proc. of 27th IAEA Fusion Energy Conf. (Ahmedabad, India) pp $\mathrm{TH} / 4-3$

[50] Del-Castillo-Negrete D, Carbajal L, Spong D A and Izzo V A 2018 Physics of Plasmas 25056104 ISSN 1070-664X URL https://doi.org/10.1063/1.5018747

[51] Tinguely R A, Granetz R S, Hoppe M and Embréus O 2018 Plasma Physics and Controlled Fusion 60124001 URL https://doi.org/10.1088/1361-6587/aae6ba\{\%\}0A

[52] Hoppe M, Embréus O, Tinguely R A, Granetz R S, Stahl A and Fülöp T 2018 Nuclear Fusion 58 ISSN 17414326 (Preprint 1709.00674) URL https://doi.org/10.1088/1741-4326/aa9abb

[53] Carbajal L, Del-Castillo-Negrete D, Spong D A, Seal S and Baylor L R 2017 Physics of Plasmas 24042512 ISSN 1070-664X URL http://dx.doi.org/10.1063/1.4981209

[54] Spong D A, Heidbrink W, Paz-Soldan C, Du X, Thome K E, Van Zeeland M, Collins C S, Lvovskiy A, Moyer R A, Austin M E, Brennan D, Liu C, Jaeger E and Lau C 2018 Physical Review Letters 120155002 ISSN 0031-9007 URL https://link.aps.org/doi/10.1103/PhysRevLett.120. 155002

[55] Connor J W and Hastie R J 1975 Nuclear Fusion 15415 URL https://doi.org/10.1088/ $0029-5515 / 15 / 3 / 007$ 
[56] Granetz R S, Esposito B, Kim J H, Koslowski H R, Lehnen M, Martin-Solis J R, Paz-Soldan C, Rhee T, Wesley J C, Zeng L and Group I M 2014 Physics of Plasmas 21072506 URL http://dx.doi.org/10.1063/1.4886802

[57] McDevitt C J, Guo Z and Tang X 2018 Plasma Physics and Controlled Fusion 60024004 ISSN 13616587 URL https://doi.org/10.1088/1361-6587/aa9b3f

[58] Heidbrink W W, Paz-Soldan C, Spong D A, Du X D, Thome K E, Austin M E, Lvovskiy A, Moyer R A, Pinsker R I and Van Zeeland M A 2019 Plasma Physics and Controlled Fusion 61014007 URL https://doi .org/10.1088/1361-6587/aae2da

[59] Jaeger E F, Berry L A, D'Azevedo E, Batchelor D B and Carter M D 2001 Physics of Plasmas 8 1573-1583 ISSN 1070664X URL https://doi.org/10.1063/1.1359516

[60] Liu C, Hirvijoki E, Fu G Y, Brennan D, Bhattacharjee A and Paz-Soldan C 2018 Physical Review Letters 120265001 ISSN 1079-7114 URL https://doi.org/10.1103/PhysRevLett. 120.265001

[61] Aleynikov P B and Breizman B N 2015 Nuclear Fusion 55043014 ISSN 0029-5515 URL https://doi.org/10.1088/0029-5515/55/4/043014

[62] Liu C, Shi L, Hirvijoki E, Brennan D, Bhattacharjee A, Paz-Soldan C and Austin M E 2018 Nuclear Fusion 58096030 (Preprint 1803.09897) URL https://doi.org/10.1088/1741-4326/aacc9b

[63] Paz-Soldan C, La Haye R J, Shiraki D, Buttery R J, Eidietis N W, Hollmann E M, Moyer R A, Boom J E and Chapman I T 2016 Nuclear Fusion 56056010 ISSN 0029-5515 URL https://doi.org/10.1088/0029-5515/56/5/056010

[64] Shiraki D, Commaux N, Baylor L R, Cooper C M, Eidietis N W, Paz-Soldan C, Combs S K and Meitner S J 2018 Nuclear Fusion 58056006 URL https://doi.org/10.1088/1741-4326/ aab0d6

[65] Parail V V and Pogutse O P 1978 Nuclear Fusion 18303 URL http://iopscience.iop.org/ $0029-5515 / 18 / 3 / 001$

[66] Zhou R J, Hu L Q, Li E Z, Xu M, Zhong G Q, Xu L Q, Lin S Y and Zhang J Z 2013 Plasma Physics and Controlled Fusion 55055006 ISSN 0741-3335 URL https://doi.org/10.1088/ $0741-3335 / 55 / 5 / 055006$

[67] Hollmann E M, Bykov V and Moyer R A 2019 Nuclear Fusion (in preparation)

[68] Eidietis N W, Commaux N, Hollmann E M, Humphreys D A, Jernigan T C, Moyer R a, Strait E J, Van Zeeland M A, Wesley J C and Yu J H 2012 Physics of Plasmas 19056109 ISSN 1070664X URL http://dx.doi.org/10.1063/1.3695000

[69] Papp G and al E 2016 Proc. of 26th IAEA Fusion Energy Conf. (Kyoto, Japan) EX/94 URL https://nucleus.iaea.org/sites/fusionportal/SharedDocuments/FEC2016/ fec2016-preprints/preprint0502.pdf

[70] Mlynar J, Ficker O, Macusova E, Markovic T, Naydenkova D, Papp G, Urban J, Vlainic M, Vondracek P, Weinzettl V, Bogar O, Bren D, Carnevale D, Casolari A, Cerovsky J, Farnik M, Gobbin M, Gospodarczyk M, Hron M, Kulhanek P, Havlicek J, Havranek A, Imrisek M, Jakubowski M, Lamas N, Linhart V, Malinowski K, Marcisovsky M, Matveeva E, Panek R, Plyusnin V V, Rabinski M, Svoboda V, Svihra P, Varju J, Zebrowski J, Team t C T and the EUROfusion MST1 2019 Plasma Physics and Controlled Fusion 61014010 ISSN 0741-3335 URL https://doi.org/10.1088/1361-6587/aae04a

[71] Khayrutdinov R R and Lukash V E 1993 Journal of Computational Physics 109 193-201 URL https://doi.org/10.1006/jcph.1993.1211

[72] Konovalov S V 2016 Assessment of the runaway electron energy dissipation in ITER Proc. of 26th IAEA Fusion Energy Conf. (Kyoto, Japan) pp TH/7-1 URL https://conferences .iaea.org/ indico/event/98/contributions/11966/

[73] Kiramov D I and Breizman B N 2017 Physics of Plasmas 24100702 ISSN 1070-664X URL http://dx.doi.org/10.1063/1.4993071

[74] Kiramov D I and Breizman B N 2018 Physics of Plasmas 25092501 ISSN 10897674 URL https://doi.org/10.1063/1.5046517 
[75] Humphreys D A and Kellman A G 1999 Physics of Plasmas 62742 ISSN 1070664X URL http://dx.doi.org/10.1063/1.873231

[76] Martin-Solis J R, Alvarez J D, Sanchez R and Esposito B 1998 Physics of Plasmas 52370 ISSN 1070664X URL http://scitation.aip.org/content/aip/journal/pop/5/6/10.1063/ 1.872911

[77] Martin-Solis J R, Loarte A and Lehnen M 2017 Nuclear Fusion 57066025 URL https://doi. org/10.1088/1741-4326/aa6939

[78] Paz-Soldan C, Eidietis N W, Liu Y, Shiraki D, Boozer A H, Hollmann E M, Kim C C and Lvovskiy A 2019 Plasma Physics and Controlled Fusion (in press) 61 12400X URL https: //doi.org/10.1088/1361-6587/aafd15

[79] Aleynikova K, Huijsmans G T A and Aleynikov P B 2016 Plasma Physics Reports 42 486-494 ISSN 1063-780X URL http://link.springer.com/10.1134/S1063780X16050019

[80] Loarte A, Riccardo V, Martin-Solis J R, Paley J, Huber A, Lehnen M and Contributors J E 2011 Nuclear Fusion 5173004 URL https://doi.org/10.1088/0029-5515/51/7/073004

[81] Zeng L, Chen Z Y, Dong Y B, Koslowski H R, Liang Y, Zhang Y P, Zhuang H D, Huang D W and Gao X 2017 Nuclear Fusion 57046001 ISSN 17414326 URL https://doi.org/10.1088/ 1741-4326/aa57d9

[82] Chu N, Sun Y, Gu S, Wang H H, Hu Y J, Shi T H and Chen D L 2018 Nuclear Fusion 58104004 URL https://doi .org/10.1088/1741-4326/aad70c

[83] Fasoli A, Testa D, Panis T, Klein A, Snipes J A, Sears J, Gryaznevich M, Martin R and Pinches S D 2010 Plasma Physics and Controlled Fusion 52 ISSN 07413335 URL https: //dx.doi.org/10.1088/0741-3335/52/7/075015

[84] Guo Z, McDevitt C J and Tang X 2018 Physics of Plasmas 25032504 ISSN 1070-664X URL https://doi.org/10.1063/1.5019381 